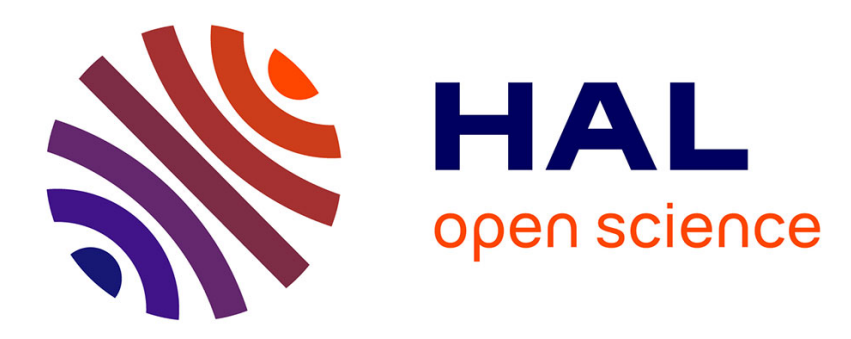

\title{
Optimal Prevention and Elimination of Infectious Diseases
}

Hippolyte d'Albis, Emmanuelle Augeraud-Véron

\section{To cite this version:}

Hippolyte d'Albis, Emmanuelle Augeraud-Véron. Optimal Prevention and Elimination of Infectious Diseases. 2021. halshs-02563356v2

\section{HAL Id: halshs-02563356 \\ https://shs.hal.science/halshs-02563356v2}

Preprint submitted on 19 Jan 2021

HAL is a multi-disciplinary open access archive for the deposit and dissemination of scientific research documents, whether they are published or not. The documents may come from teaching and research institutions in France or abroad, or from public or private research centers.
L'archive ouverte pluridisciplinaire HAL, est destinée au dépôt et à la diffusion de documents scientifiques de niveau recherche, publiés ou non, émanant des établissements d'enseignement et de recherche français ou étrangers, des laboratoires publics ou privés. 


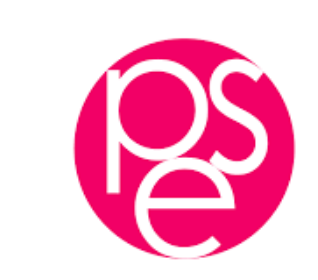

PARISSCHOOL OFECONOMICS
ECOLED'ECONOMIEDE PARIS

WORKING PAPER Nº $2020-21$

Optimal Prevention and Elimination of Infectious Diseases

Hippolyte d'Albis

Emmanuelle Augeraud-Véron

JEL Codes: I18, C61, E13

Keywords: Infectious Diseases, Optimal Control.

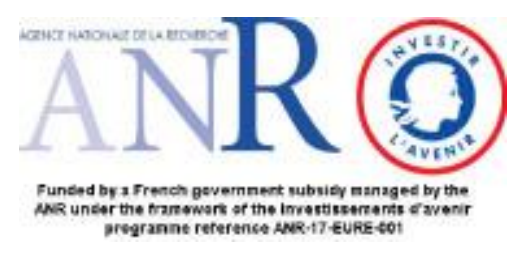




\title{
Optimal Prevention and Elimination of Infectious Diseases ${ }^{1}$
}

\author{
Hippolyte d'ALBIS ${ }^{2}$ \\ Paris School of Economics, CNRS \\ Emmanuelle AUGERAUD-VÉRON ${ }^{3}$ \\ GREThA, University of Bordeaux
}

January 18, 2021

\footnotetext{
${ }^{1}$ We are grateful to Andrés Carvajal and the Special Editors of this issue, two anonymous referees, and to Jean-Pierre Amigues, Eric Benoît, Martial Dupaigne, Albert Marcet and participants in seminars at Universities of Exeter, Paris and Toulouse, at European University Institute and ETH Zurich for their valuable comments and suggestions. The usual disclamer applies.

${ }^{2}$ Correspondence: 48 boulevard Jourdan, 75014 Paris, France. E-mail: hdalbis@psemail.eu

${ }^{3}$ Correspondence: 6 avenue Léon Duguit, 33600 Pessac, France. E-mail: emmanuelle.augeraud@u-bordeaux.fr
} 


\begin{abstract}
This article studies the optimal intertemporal allocation of resources devoted to the prevention of deterministic infectious diseases that admit an endemic steadystate. Under general assumptions, the optimal control problem is shown to be formally similar to an optimal growth model with endogenous discounting. The optimal dynamics then depends on the interplay between the epidemiological characteristics of the disease, the labour productivity and the degree of intergenerational equity. Phase diagrams analysis reveal that multiple trajectories, which converge to endemic steady-states with or without prevention or to the elimination of the disease, are feasible. Elimination implies initially a larger prevention than in other trajectories, but after a finite date, prevention is equal to zero. This "sooner-the-better" strategy is shown to be optimal if the pure discount rate is sufficiently low.
\end{abstract}

JEL Classification: I18, C61, E13

Keywords: Infectious Diseases, Optimal Control. 


\section{Introduction}

Infectious diseases constitute major health issues for which there is large consensus on the legitimacy of governments interventions. Yet few analysis has been undertaken to determine the socially optimal allocation of resources to control the evolution of the disease. In this article, we derive a welfare criterion from individual preferences and give precise foundations on a generally held belief, according to which intervention should begin as soon as possible and involve large expenses. We focus our analysis on expenditure that reduce the number of contacts relevant for infection transmission per unit of time and, consequently, reduce the spread of the disease. They include prevention campaigns that modify individuals' behaviors, by e.g. the diffusion of masks or condoms that reduce the probability of a contact to be infectious, and any measures that reduce physical contacts such as lockdowns of populations. Throughout the article, all those policies will be named as prevention measures, and the aim will be to determine the optimal intensity over time of the prevention in a economy that faces an infectious disease.

Infectious disease' prevention is a legitimate topic for economics since, as argued by Bloom and Canning [10], there is little doubt that resource constraints play an important role in the spread of the disease. Moreover, as other diseases, they importantly affect labor and capital markets, and thus growth. However, and despite the fact that past diseases were recognized as economic tragedies, 
Gersovitz and Hammer [28] pointed out that it is only since the 1990's that economists have entered the field. The interest has skyrocketed recently with the Covid-19 outbreak. Most articles in the literature adopt a positive approach focusing on private behaviors, such as individual choices on self-exposure to the risk (as in Geoffard and Philipson [30] and Kremer [39]), health expenditure (Momota et al. [42]), human capital accumulation (Bell and Gersbach [6], Corrigan et al. [20] and Boucekkine et al. [13]), fertility (Young [51]), income distribution ([14]) or biodiversity (Bosi and Desmarchelier [11]). Some papers analyzes the effect of public policies on private behavior (e.g. Geoffard and Philipson [31]), while others consider optimal policy correcting for the obvious externalities produced by infectious diseases (e.g. Gersovitz and Hammer [29], Francis [25], Gersovitz [27], Bethune et al. [9] or Eichenbaum et al. [23]).

While few papers in economics adopt a normative perspective, one observes there exists an abundant mathematical epidemiology literature, which dates back from Bernouilli [8]! Following Sethi [47] and Wickwire [50], it is common to use optimal control techniques to define the desirable timing of vaccination, screening or health promotion campaigns. In most cases these studies use as a criterion a convex combination of the dynamic costs of the control and of the number of infected individuals. Moreover, the time horizon is usually finite and, in analytical models, the problem is linear with respect to the control. Based on this approach Behncke [7] finds that the optimal control of an epidemics is, in general, such that the prevention effort is maximal on some initial time 
interval and then set to zero (see also Buonomo et al. [17] Morris et al. [43]). When the case of disease elimination is considered, the problem is more complex since terminal conditions are free (Barrett and Hoel [5]). Some economic studies (in particular Gersovitz and Hammer [28], Francis [25], Feichtinger et al. [24], Alvares and al. [2] or Rowthorn and Toxvaerd [46]) have used a slightly more sophisticated criterion as they consider the present discounted value of total income net of the costs of the disease and of the control.

We propose an optimal control model in the tradition of Ramsey [45], Cass [18] and Koopmans [38] in which the whole population is affected by an infectious disease, whose dynamics is rather general while admitting an endemic steadystate. The social welfare function is the present discounted value of the product of individual utility and the size of the population. We notably show how this criteria relies on preferences. The introduction of population in the objective is key to avoid the problem stressed by the optimal population literature (see notably Dasgupta [21]) about maximizing the welfare of alive individuals only. By considering an endogenous population in the welfare function, our work distinguishes itself and extends important theoretical contributions of Delfino and Simmons [22], Boucekkine et al. [12], Goenka and Liu [32] or Goenka et al. [34], who in most cases consider specific epidemiological processes and study local dynamics. It also complement works such as those of Gersovitz and Hammer [29] or Eichenbaum et al. [23], who proceed by simulations.

A second important assumption we make is about the production structure 
which is of pure exchange, i.e. without physical capital, which allow us to completely characterize the model despite a general formulation for the dynamics of the infectious disease. The dynamics of the optimal prevention then depends on the interplay between the epidemiological characteristics of the disease, labour productivity and intergenerational equity. We believe it's important to characterize the global dynamics of the optimal system as it permits to visualize both the objective that should be reached in the short run, and some trajectories that could be hidden while performing local long-run analysis.

We find that it may be optimal to reduce the prevalence rate of the infectious disease in the long run only if labour productivity is above some minimal level. If this threshold is not reached, prevention is then at best temporary, simply slowing down the spread of the infectious disease. However, it may not be optimal to undertake temporary prevention. When instead labour productivity is sufficiently high, permanent allocation of resources to prevention is feasible though not necessarily optimal. If permanent prevention is socially optimal, the prevention effort monotonically increases with time for low initial prevalence rate, and is hump-shaped or decreasing otherwise. Hence, our paper establishes that under a welfare criterion for social intertemporal optimization a "soonerthe-better" strategy may not be the optimal one, in contrast to Behncke [7]. This statement is however reversed when we consider paths that may yield to the elimination of the infectious disease. We first show that a simple modification of usual deterministic models describing endemic infectious diseases can be made 
to allow for elimination in finite time. ${ }^{1}$ Trajectories that drive to elimination are characterized by an increasing prevention for a finite interval of time and, once the infectious disease is eliminated, the prevention is zero. We show that upon existence, such paths are optimal if the pure discount rate is sufficiently small. In that case, it consequently is socially desirable that prevention should begin as soon as possible. Moreover, the effort initially devoted to prevention is larger for elimination than for any other dynamics. This is a theoretical foundation for a "whatever it costs" response to a disease outbreak.

We begin by presenting the dynamics of the population affected by an infectious disease in section 2. The epidemiological assumptions are put forward and discussed using standard examples of HIV and flu. In section 3 we set up the social planner's problem, then prove the existence of a solution and characterize it. The dynamics of the optimal prevention is analyzed in section 4 . The question on whether it is socially optimal to eliminated the infectious disease is studied section 5, and illustrated with a hand solved example. Section 6 concludes.

\section{Dynamics of the infectious disease}

This section presents the general characterization of the dynamics of the disease that encompasses a number of models proposed in the epidemiological literature. It's a two-compartment model in which the total population is allowed

\footnotetext{
${ }^{1}$ Note that stochastic models are usually considered to explain why finite time elimination of the disease is possible (Britton [16], Allen and Lahodny [1]). Contrary to what happens with deterministic models, it is there possible that the disease never breaks out when a small number of infectives are initially present in the whole population, even if the reproduction number is greater that one.
} 
to change with the prevalence rate. The evolution of the disease is constrained by some assumptions that are satisfied, for instance, in classical compartmental models (Kermack and Mac Kendrick [37]). We also propose to introduce an arbitrarily small threshold below which the disease is considered as eliminated. This modelling feature will be key in the subsequent analysis.

\subsection{A two-class framework}

Time is continuous and indexed by $t \in \mathbb{R}^{+}$. The population at time $t$, whose size is denoted $P_{t}$, is affected by an infectious disease and is thus decomposed in two classes of individuals: the susceptible, who are healthy, and the infected, who may transmit the disease. The number of individual of each class is respectively denoted $S_{t}>0$ and $I_{t} \geq 0$, and satisfy: $P_{t}=S_{t}+I_{t}$. It will be convenient to define the relative share of infected individuals with respect to share of susceptible ones as follows: $a_{t}=I_{t} / S_{t}$. This ratio is a monotonically increasing transformation of the traditional prevalence, given by: $I_{t} / P_{t}=a_{t} /\left(1+a_{t}\right)$, and will be named as the prevalence index throughout the remaining of the paper. Moreover, we consider the following general law of motion for $a_{t}$ :

$$
\dot{a}_{t}=g\left(h_{t}, a_{t}\right) a_{t},
$$

where the dot indicates the first derivative with respect to time and where $h_{t}$ stands for the per capita expenditure devoted to the disease's control. These expenditure can be interpreted as prevention campaigns and any preventive measures that reduce the number of contacts relevant for infection transmission 
per unit of time and, consequently, reduce the spread of the disease (see, for instance, Castilho [19]). Note that these expenditure can not stand for vaccination campaigns or screening and isolation strategies, as they produce a third class of individuals within the population; a case that is not considered here. In the rest of the paper, $h_{t}$ will be referred as the level of prevention at time $t$.

The growth rate of the prevalence index, characterized by function $g$, is supposed to satisfy:

H1. $g: \mathbb{R}_{+}^{2} \rightarrow \mathbb{R}$, is $\mathcal{C}^{2}, g_{1}^{\prime}\left(h_{t}, a_{t}\right)<0, g_{2}^{\prime}\left(h_{t}, a_{t}\right)<0$ and $g_{11}^{\prime \prime}\left(h_{t}, a_{t}\right)>0$. There exist $a^{*}>0$ and $h^{*}>0$ such that $g\left(0, a^{*}\right)=g\left(h^{*}, 0\right)=0$.

Assumption $\mathrm{H} 1$ fully describes the kind of disease we are dealing with. The growth rate of the prevalence index is supposed to decrease with $a_{t}$ which, of course, does not imply a monotonic relationship between $\dot{a}_{t}$ and $a_{t}$. If there is no prevention, the disease lasts forever but stabilizes within the population: the prevalence index converges to the endemic steady-state $a^{*}$. The other steadystate, given for $a=0$, and named in the epidemiological literature the diseasefree equilibrium, can easily be check as being unstable as Assumption $\mathrm{H} 1$ implies $g(0,0)>0$. The reproduction number ${ }^{2}$, i.e. the number of secondary cases that can appears when one infected individual is added in a population of fully susceptible individuals for the duration of her illness, denoted $\mathcal{R}_{0}$, is here larger

\footnotetext{
${ }^{2}$ Formally, we can write: $a g(h, a)=F(h, a)+V(a)$ where $F$ include the terms related to the transmission of the disease and $V$ those related to other causes (death, etc.). The derivative at $a=0$ of the pervious equation is given by: $g(h, 0)=F^{\prime}(h, 0)+V^{\prime}(0)$. Then the (controled) reproduction number is defined as $\mathcal{R}_{c}=-F^{\prime}(h, 0) / V^{\prime}(0)$, while the (classical) reproduction number is $\mathcal{R}_{0}=-F^{\prime}(0,0) / V^{\prime}(0)$.
} 
than 1 (van den Driessche [49]). Figure 1a represents this dynamics.

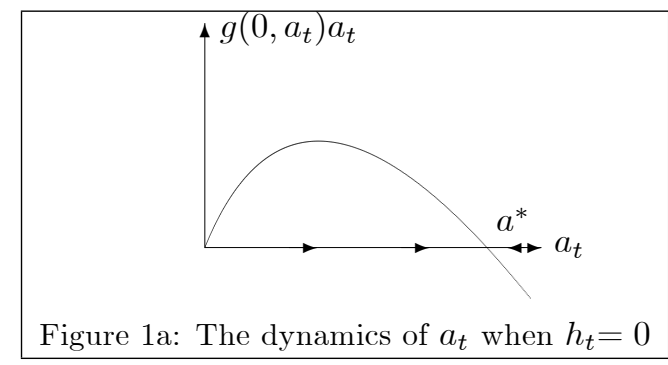

Prevention may modify the dynamics of the disease. To fix ideas, let assume that $h_{t}$ is an exogenous constant. If this constant belongs to $\left(0, h^{*}\right)$, the prevalence index converges to another stable steady-state characterized by a prevalence rate that is lower than $a^{*}$; the reproduction number with positive control $\mathcal{R}_{c}$ remains larger than 1 . However, if the constant is larger than $h^{*}$, the disease dynamics are dramatically modified as the prevalence index converges to zero; the control is sufficient to eliminate the disease in the long run and we now have $\mathcal{R}_{c}<1$. Figure 1b illustrates such dynamics with two different exogenous $h_{t}: h_{1} \in\left(0, h^{*}\right)$ and $h_{2}>h^{*}$. In the remaining of the paper, $h_{t}$ will be the control variable optimally chosen by a benevolent social planner.

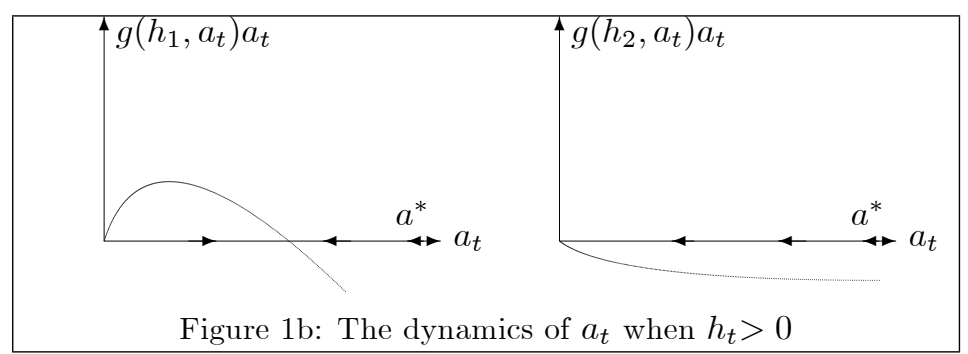

Let us remark that it is easy to extend our approach to other kinds of disease 
dynamics. An alternative dynamics without prevention could be obtained by assuming: $a^{*}<0$. Such an disease would not exhibit an endemic steadystate. The unique stable steady-state being the one with a zero prevalence index. Prevention may then be used to accelerate the convergence process. Moreover, assuming that $a^{*} \rightarrow+\infty$, allow to consider a disease whose prevalence indefinitely increases if there is no control of it.

By definition, the population growth rate writes as follows:

$$
\frac{\dot{P}_{t}}{P_{t}}=\frac{1}{1+a_{t}}\left(\frac{\dot{S}_{t}}{S_{t}}+\frac{\dot{I}_{t}}{I_{t}} a_{t}\right) .
$$

For computational reasons, we assume that the growth rate is characterized by a function denoted $n($.$) that satisfies:$

H2. $\dot{P}_{t} / P_{t}=n\left(a_{t}\right) . n: \mathbb{R}_{+} \rightarrow[\underline{n}, n(0)]$, is $C^{2}, n^{\prime}\left(a_{t}\right) \leq 0$.

Assuming that the population growth rate does not depend on $\left(S_{t}, I_{t}, h_{t}\right)$ is rather strong but is widely used in the epidemiological literature, notably in the examples presented below. Moreover, the assumption concerning non positivity of $n^{\prime}\left(a_{t}\right)$ is not only more realistic -infectious diseases increase the death ratesbut also, as it will be discussed throughout the paper, more meaningful.

\subsection{A minimal threshold for infectious diseases}

We now consider a simple generalization of equation (1), which is given by:

$$
\dot{a}_{t}=\mid \begin{array}{ll}
g\left(h_{t}, a_{t}\right) a_{t} & \text { if } a_{t}>a_{\min } \\
0 & \text { if } a_{t} \leq a_{\min },
\end{array}
$$


where $0 \leq a_{\min } \ll 1$. Equation (3) introduces a threshold below which the infectious disease is considered as eliminated. The threshold can be arbitrarily low and below it the infectious disease can not survive and spread within the population. Above this threshold, the dynamics has the same qualitative property as the one we have considered before (Assumption H1 is left unchanged). Technically, the main difference between the case $a_{\min }>0$ and the case $a_{\min }=0$ that is usually considered in the epidemiology literature, is that steady-state $a_{\min }$ can be reached in finite time, which will appear as a key property below. We will then assume that when $a_{\min }$ is reached, the disease is eliminated.

The dynamics of $a_{t}$ is represented in Figure 2, in the case of no prevention.

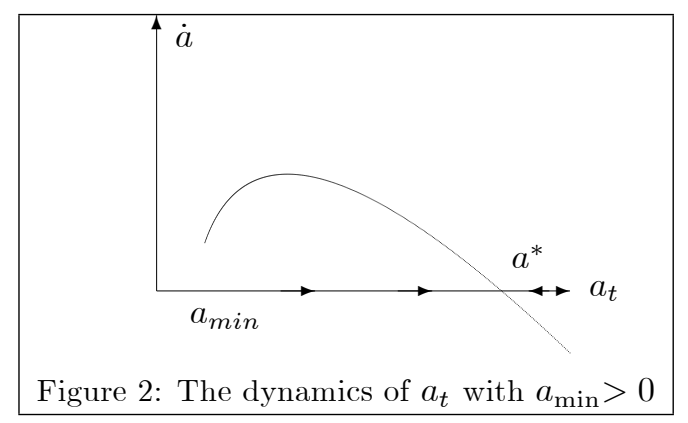

Our framework generalizes most works in economics that have analytically studied the optimal dynamics of an infectious disease: Delfino and Simmons [22], Gersovitz and Hammer [28] and Barrett and Hoel [5] study a dynamics similar to (1), but for a function $g$ which is specified, while Boucekkine et al. [12] consider a shock on the initial stock of the population describing an infectious disease with instantaneous effects and no endemic steady-state. 


\subsection{Examples}

Our assumptions on functions $g$ and $n$ are now confronted to two examples presented e.g. in Zhou and Hethcote [52]: a SI model, which can describe the HIV (similar to May and Anderson [40]) and a SIS model, which can describe a flu. In SIS models, infected individuals may recover for the disease but are not immunized and may again be infected. This is relevant for flu if one considers a mutating virus. However, most current models of the SARS-CoV-2 assume this is not the case and use three-compartments models of the SIR type (Avery et al. [4]). Such models can be covered by our analysis provided that the size of the population is kept constant.

\section{Example 1. SI model (HIV)}

Let us first suppose that $a_{\mathrm{min}}=0$ and ignore the possibility to control the disease. The natural growth rate of the susceptible population is given by $\beta-\mu$, where $\beta>0$ and $\mu>0$ respectively stand for the birth and the death rates, while the growth rate of the infected population is $\beta-\mu-\gamma$; parameter $\gamma \geq 0$ measures the over-mortality yield by the disease. Both vertical and horizontal transmission are considered: first a proportion $\pi \in[0,1]$ of the children of infected people are born healthy, while the others are infected. Moreover, as in May and Anderson [40], it is assumed that the transmission is given by a frequency-dependent transmission function: transmission is proportional, up to a parameter $\sigma>0$, to the density of infected individuals in the total population. 
The dynamics of each subpopulation is therefore given by:

$$
\begin{aligned}
\dot{S}_{t} & =(\beta-\mu) S_{t}+\beta \pi I_{t}-\sigma S_{t} \frac{I_{t}}{S_{t}+I_{t}}, \\
\dot{I}_{t} & =[\beta(1-\pi)-\mu-\gamma] I_{t}+\sigma S_{t} \frac{I_{t}}{S_{t}+I_{t}} .
\end{aligned}
$$

It can be easily shown that this system does not generically admit a steady-state except: $S_{t}=I_{t}=0$. Hence, the dynamics of the disease is better understood using per capita variables or, equivalently, by using our prevalence index $a_{t}$ (defined as $a_{t}=I_{t} / S_{t}$ ). Using (4) and (5), the dynamics of $a_{t}$ solves:

$$
\dot{a}_{t}=\left[\sigma-\beta \pi-\gamma-\beta \pi a_{t}\right] a_{t},
$$

which is a logistic equation. Therefore, if $\pi \in(0,1]$, the dynamics of $a_{t}$ writes:

$$
a_{t}=\frac{\frac{(\sigma-\beta \pi-\gamma)}{\beta \pi} a_{0}}{a_{0}+\left(\frac{(\sigma-\beta \pi-\gamma)}{\beta \pi}-a_{0}\right) e^{-(\sigma-\beta \pi-\gamma) t}} .
$$

Equation (6) admits (i) two steady-states if $\sigma>\beta \pi+\gamma$ : namely, $\hat{a}=0$, which is unstable and $a^{*}=(\sigma-\beta \pi-\gamma) / \beta \pi$, which is stable, (ii) one steady-state if $\sigma \leq \beta \pi+\gamma$ : namely, $\hat{a}=0$, which is stable. Consequently, if the transmission coefficient $\sigma$ is sufficiently low, the infectious disease ultimately disappears. Conversely, if $\sigma$ is high, the disease survives as the prevalence index stabilizes. ${ }^{3}$ If there is full vertical transmission (i.e. if $\pi=0$ ), the only possible steady state is $\hat{a}=0$, whose stability is given by the sign of $\sigma-\gamma$.

\footnotetext{
${ }^{3}$ Alternatively, one may present the stability results using the reproduction number which is here given by $\mathcal{R}_{0}=(\sigma-\beta \pi) / \gamma$. The dynamics of $a$ can be rewritten as:

$$
\dot{a}_{t}=\left[\mathcal{R}_{0}-1-\frac{\beta \pi}{\gamma} a_{t}\right] a_{t} \gamma
$$
}

which implies that $a^{*}>0$ if and only if $\mathcal{R}_{0}>1$. 
Using (4) and (5), the population growth rate $n\left(a_{t}\right)$ solves:

$$
n\left(a_{t}\right)=\beta-\mu-\frac{\gamma a_{t}}{1+a_{t}},
$$

which decreases in $a_{t}$; moreover, $\beta-\mu-\gamma \leq n\left(a_{t}\right)<\beta-\mu$. The higher bound for $n($.$) being the population growth rate without infectious disease.$

Suppose now that prevention may affect the disease dynamics through the transmission parameter by reducing the number of effective contacts by time unit. Let $h_{t}$ be the per capita expenditure devoted to prevention and the transmission coefficient at time $t$ be a function that writes $\sigma\left(h_{t}\right)$ and satisfies $\sigma^{\prime}\left(h_{t}\right)<0$. The dynamics of $a_{t}$ is now given by:

$$
\dot{a}_{t}=\left[\sigma\left(h_{t}\right)-\beta \pi-\gamma-\beta \pi a_{t}\right] a_{t} .
$$

It is immediate to check that Assumption H1 is satisfied only if $\pi>0$ and if $\sigma(0)>\beta \pi+\gamma$ and that Assumption $\mathrm{H} 2$ is always satisfied. Hence, full vertical transmission is excluded here.

Remarkably, our example easily extends to the case $a_{\min }>0$, for which equation (7) holds for $a_{t} \geq a_{\min }$ only. The stability analysis is modified as follows: if $\sigma>\beta \pi+\gamma, a^{*}$ remains a stable steady-state while if $\sigma<\beta \pi+\gamma$, $a_{\min }$ is stable and can be reached in finite time.

\section{Example 2. SIS model (Flu)}

Following Hethcote and Yorke [36], let us now consider the following dynam- 
ics for the susceptible and the infected:

$$
\begin{aligned}
\dot{S}_{t} & =\beta\left(S_{t}+I_{t}\right)-\mu S_{t}+\delta I_{t}-\sigma S_{t} \frac{I_{t}}{S_{t}+I_{t}}, \\
\dot{I}_{t} & =-(\delta+\mu+\gamma) I_{t}+\sigma S_{t} \frac{I_{t}}{S_{t}+I_{t}} .
\end{aligned}
$$

The main difference are that there is no vertical transmission of the disease, and that infected individuals recover at rate $\delta>0$. Then, the dynamics of the prevalence index satisfies:

$$
\dot{a}_{t}=\left[\sigma-\delta-\gamma-\beta-a_{t}(\delta+\beta)\right] a_{t},
$$

while the population growth rate is the same as (8). The stability analysis is similar to that of the previous example. ${ }^{4}$ Again, we can assume that $\sigma$ is a decreasing function of the level of prevention $h$.

\section{The optimal control problem}

This section establishes the optimal control problem we are going to study. It is an infinite horizon framework with an economic structure and the population dynamics described in section 2 . We first present and discusses the social welfare function and then prove the existence of an optimal solution.

\subsection{The social welfare function}

The social welfare function we introduce is derived from the aggregation of individual's preferences. Each individual is supposed to belong to a dynasty

\footnotetext{
${ }^{4}$ The reproduction number is now given by $\mathcal{R}_{0}=\sigma /(\delta+\beta+\gamma)$ and the dynamics of the prevalence index can be rewritten as:

$$
\dot{a}_{t}=(\delta+\gamma+\mu)\left[\mathcal{R}_{0}-1-a_{t} \frac{\delta+\beta}{\delta+\gamma+\mu}\right] a_{t} .
$$
}


of altruistic individuals. Without infectious diseases, the growth rate of the dynasty is equal to $n(0)$. However, at each point of time, members of a given dynasty may die from the disease, which reduces the growth rate of the dynasty. We denote by $\lambda_{t}$ the probability as of time $t=0$ that the dynasty is still alive at time $t$. If alive at time $t$, the utility of a dynasty member depends on consumption $c_{t}$ and is independent of the health status. The utility function is denoted $u\left(c_{t}\right)$. If not alive at time $t$, the utility is supposed to be a constant that is normalized to the value of the utility with non consumption: $u(0)$. Importantly, we suppose that $u(0) \gg-\infty$. The expected utility of the dynasty at time $t=0$ is therefore:

$$
\int_{0}^{+\infty} e^{-(\rho-n(0)) t}\left[\lambda_{t} u\left(c_{t}\right)+\left(1-\lambda_{t}\right) u(0)\right] d t
$$

where $\rho$ is the pure discount rate which satisfies the following restriction:

H3. $\rho>n(0)$.

Moreover, function $u$ satisfies the following assumption:

H4. $u: \mathbb{R}_{+} \rightarrow \mathbb{R}_{+}, u \in C^{3}, u^{\prime}>0, u^{\prime \prime}<0$ and $\lim _{c \rightarrow 0} u^{\prime}(c)=+\infty$.

To obtain the social welfare function, we assume that the population is composed by a continuum of identical dynasties, whose total size at time $t=0$ is $P_{0}$. By the law of large numbers, the probability $\lambda_{t}$ is, at the aggregate level, the ratio between the size of the population and the size that would prevail in the absence of infection. Thus: $\lambda_{t}=P_{t} / P_{0} e^{n(0) t}=e^{\int_{0}^{t}\left[n\left(a_{s}\right)-n(0)\right] d s}$. The social 
welfare function at time $t=0$ is therefore simply obtained by multiplying the function (13) by the initial size of the population $P_{0}$, and rearranging to obtain:

$$
P_{0} \int_{0}^{+\infty} e^{-\int_{0}^{t}\left[\rho-n\left(a_{s}\right)\right] d s}\left[u\left(c_{t}\right)-u(0)\right] d t+\frac{P_{0} u(0)}{\rho-n(0)} .
$$

Maximizing this latter function is, in fact, equivalent to maximizing:

$$
\int_{0}^{\infty} e^{-\rho t} P_{t} u\left(c_{t}\right) d t
$$

The social planner function is thus the discounted value of the product of the size of the population, $P_{t}$, and of the instantaneous utility of each individual. In this total utilitarism (i.e. Bentamite) function, we observe that for a given path of consumption, a larger population increases the social welfare. Consequently, the assumption $n^{\prime}\left(a_{t}\right)<0$, implies that reducing the number of infected individuals increases welfare, everything being equal. Observe finally that the limit case $n^{\prime}\left(a_{t}\right)=0$, which features infectious diseases that have no impact on the population growth, implies $\lambda_{t}=1$ and a standard objective function. We notice that most theoretical works (by most notably Boucekkine et al. [12], Goenka and Liu [32], Goenka et al. [34] or Rowthorn and Toxvaerd [46]) consider either that the infectious disease has no impact on population growth or that the population does not enter the social welfare function. Delfino and Simmons [22] is an exception as they assume that the disease dynamics impact the instantaneous utility but they do not provide a theoretical foundation for their specification. 


\subsection{The social planner's program}

The social planner faces the resource constraint of the economy. There is one material good produced using labor and it is assumed that the productivity of an infected individual is lower than the one of an susceptible individual. Production per capita is assumed to be a function of prevalence that is written as $\alpha f\left(a_{t}\right)$ where $\alpha$ is a measure of the productivity of labor and $f$ is a measure of the quantity of labor. It is assumed that labor is a decreasing and concave function of prevalence: $f()>0,. f^{\prime}()<$.0 and $f^{\prime \prime}() \leq$.0 . The produced good can be used for consumption or for the expenditure devoted to the control of the infectious disease. The resource constraint written in per capita units is therefore:

$$
c_{t}+h_{t}=\alpha f\left(a_{t}\right)
$$

Moreover, consumption, prevention and the prevalence index should be non negative. The program of the social planner is to maximize (15) subject to (1) and (16). It writes:

$$
\begin{array}{l|l}
\max _{h_{t}} \int_{0}^{\infty} e^{-\int_{0}^{t} \theta\left(a_{s}\right) d s} u\left(\alpha f\left(a_{t}\right)-h_{t}\right) d t, \\
\text { s.t. } & \dot{a}_{t}=\mid \begin{array}{ll}
g\left(h_{t}, a_{t}\right) a_{t} & \text { if } a_{t}>a_{\text {min }} \\
0 & \text { if } a_{t} \leq a_{\text {min }},
\end{array} \\
& 0 \leq h_{t} \leq \alpha f\left(a_{t}\right) \text { and } a_{0} \in\left(a_{\mathrm{min}}, a^{*}\right) \text { given. }
\end{array}
$$

where $\theta\left(a_{t}\right):=\rho-n\left(a_{t}\right)$. We note that since $n$ is a decreasing function and given Assumption H3, one has $\theta()>$.0 and $\theta^{\prime}()>$.0 . As discussed in Boucekkine et al. [15], a problem with endogenous population is formally similar to an optimal 
growth model with endogenous discounting.

To reduce the length of the proofs and to focus on the meaningful cases, we solved the problem for $a_{0} \in\left(a_{\min }, a^{*}\right)$ but the analysis can be generalized to $a_{0}>a^{*}$. Let us notice that the problem is trivial if the initial prevalence of the infectious disease is below the minimal threshold (i.e. $a_{0} \leq a_{\text {min }}$ ): the optimal consumption is equal to the production $\alpha f\left(a_{\min }\right)$ and the prevention is equal to zero.

The intertemporal trade-off is the following: an increase in $h_{t}$ yields a reduction of both the immediate per-capita consumption and the prevalence of the infectious disease. The latter implies first an increase in future per-capita production and therefore the expenditure devoted to prevention can be understood as an investment. Moreover, reducing prevalence leads to a modification of the spread between the discount rate and the population growth rate. As $\theta^{\prime}\left(a_{t}\right)>0$, an increase in $h_{t}$ implies a reduction of the spread, meaning a more equal treatment between individuals of different generations as it increases the weight associated to the utility of future generations.

\subsection{An existence result}

The program (17) is a non autonomous problem with endogenous discounting but can be equivalently analyzed as an exogenous discounting problem using the virtual time method described by Uzawa [48]. The following results are then derived. 
Lemma 1 There exists an optimal solution to program (17). The optimal solution satisfies $h_{t}<\alpha f\left(a_{t}\right)$.

Proof. See Appendix.

We now turn to the characterization of the solution by analyzing first the case such that $a_{\mathrm{min}}=0$, which is standard in mathematical epidemiology. The main goal is to characterized the dynamics of the optimal prevention strategy. Then, we turn to the case $a_{\min }>0$ to analyze the issue of elimination.

\section{Is prevention optimal?}

This section studies the system of equations (18) that characterizes the dynamics of prevalence and prevention when there is no minimal threshold for the infectious disease (i.e. for $a_{\min }=0$ ). Local dynamics around steady-state are first studied and a geometrical analysis using phase diagrams is then provided.

\subsection{The optimal dynamics for $a_{\min }=0$}

Let us first give the equations describing the optimal trajectories.

Lemma 2 An optimal path is necessarily a solution of the following system:

$$
\begin{aligned}
& \begin{cases}\dot{a}_{t}=\frac{g\left(h_{t}, a_{t}\right) a_{t}}{\theta\left(a_{t}\right)} & \text { if } h_{t}>0, \\
\dot{h}_{t}=\frac{\Phi\left(h_{t}, a_{t}, c_{t}\right)}{-\theta\left(a_{t}\right)\left[\frac{u^{\prime \prime}\left(\alpha f\left(a_{t}\right)-h_{t}\right)}{u^{\prime}\left(\alpha f\left(a_{t}\right)-h_{t}\right)}+\frac{g_{11}^{\prime \prime}\left(h_{t}, a_{t}\right)}{g_{1}^{\prime}\left(h_{t}, a_{t}\right)}\right]} & \\
\dot{a}_{t}=\frac{g\left(0, a_{t}\right) a_{t}}{\theta\left(a_{t}\right)} & \text { if } h_{t}=0,\end{cases}
\end{aligned}
$$


with $c_{t}=\alpha f\left(a_{t}\right)-h_{t}$ and:

$$
\begin{aligned}
\Phi\left(h_{t}, a_{t}, c_{t}\right)= & {\left[-\alpha f^{\prime}\left(a_{t}\right) \frac{u^{\prime \prime}\left(c_{t}\right)}{u^{\prime}\left(c_{t}\right)}+\frac{g_{12}^{\prime \prime}\left(h_{t}, a_{t}\right)}{g_{1}^{\prime}\left(h_{t}, a_{t}\right)}+\frac{\theta^{\prime}\left(a_{t}\right)}{\theta\left(a_{t}\right)}\right] g\left(h_{t}, a_{t}\right) a_{t} } \\
& +\left[-\alpha f^{\prime}\left(a_{t}\right)+\frac{u\left(c_{t}\right)}{u^{\prime}\left(c_{t}\right)} \frac{\theta^{\prime}\left(a_{t}\right)}{\theta\left(a_{t}\right)}\right] g_{1}^{\prime}\left(h_{t}, a_{t}\right) a_{t} \\
& -g_{2}^{\prime}\left(h_{t}, a_{t}\right) a_{t}+\theta\left(a_{t}\right) .
\end{aligned}
$$

Proof. See the Appendix.

From Lemma 2, it is straightforward to derive the optimal dynamics of consumption, which is always positive (see Lemma 1).

\subsection{Prevention in the long run}

Let us first consider the local dynamics in the neighborhood of the steady-states of (18). We define a 'corner steady-state' as a steady-state for which the optimal prevention is zero and an 'interior steady-state' as a steady-state for which the optimal prevention is positive.

Given Assumption H1, the pairs $\left(a^{*}, 0\right)$ and $(0,0)$ are the two corner steadystates of our system. The first one satisfies the following properties:

Lemma 3 Provided that there exists an optimal path that converges to the corner steady-state $\left(a^{*}, 0\right)$, it satisfies $h_{t}=0$ for t large enough .

Proof. See the Appendix.

Whatever its initial dynamics, the optimal prevention is thus equal to zero after a finite date if the prevalence index converges to an endemic steady-state without 
prevention. The intuition is that $\left(a^{*}, 0\right)$ is not a steady-state for the interior dynamics of system (18). Optimal prevention may hence not converge to zero but only reach zero in a finite time. Since it is never optimal to reach $a^{*}$ in a finite time, we conclude that $h_{t}=0$ in the neighborhood of $\left(a^{*}, 0\right)$. Note that this argument rules out local indeterminacy. This results confirms the initial findings of Goenka et al. [34] who provide conditions for local uniqueness in a setting in physical capital.

Concerning the second corner steady-state, we obtained the following:

Lemma 4 There is no optimal path that converges to $(0,0)$.

Proof. See the Appendix.

The disease elimination is not a possible output of models with a traditional representation of infectious diseases. The intuition, which is illustrated in the phase diagrams below is the following. A trajectory that would converges to the disease-free-equilibrium would necessary imply $\dot{h}_{t}>0$ forever, which is not possible because of the budget constraint. The same result is obtained by Rowthorn and Toxvaerd [46] in a similar setting. Below, we show how going beyond this basic statement as we obtain a trajectory leading to elimination that can be optimal.

Let us now study interior steady-states, upon which prevention is positive. Using (18), such a steady-state is a pair $(a, h)$ that satisfies $a \in\left(0, a^{*}\right), h \in$ 
$(0, \alpha f(a))$ and solves:

$$
\begin{aligned}
g(h, a) & =0 \\
-\left[g_{2}^{\prime}(h, a) a-\theta(a)\right] & =-\left[-\alpha f^{\prime}(a)+\frac{u(c)}{u^{\prime}(c)} \frac{\theta^{\prime}(a)}{\theta(a)}\right] g_{1}^{\prime}(h, a) a,
\end{aligned}
$$

where $c=\alpha f(a)-h$. Then, a necessary condition for existence of an interior steady-state is the positivity of equation (21)'s right hand side, which rewrites as follows:

$$
\frac{d}{d a}\left(\frac{u(\alpha f(a)-h)}{\theta(a)}\right)<0 .
$$

Condition (22) means that the discounted welfare of a generation in the long run should be increased by a reduction of the disease. The increase in utility implied by a marginal decreases of $a$ (measured by $-\alpha f^{\prime}(a) u^{\prime}(c) / \theta(a)$ ) should be larger than the negative impact on the endogenous discount (given by $\left.\theta^{\prime}(a) u(c) /(\theta(a))^{2}\right)$. Necessary and sufficient conditions (20) and (21) may be rewritten in the following way:

$$
\frac{u^{\prime}(u(\alpha f(a)-\eta(a)))}{\theta(a)}=\frac{d \dot{a}}{d h} \times \frac{d}{d a}\left(\frac{u(\alpha f(a)-\eta(a))}{\theta(a)}\right),
$$

where $\eta(a)$ is the implicit relation between $h$ and $a$ derived from Assumption H1. The contemporaneous desutility induced by a marginal increase in prevention should equal the benefits of the reduction of the prevalence of the disease.

The following lemma studies the existence of interior steady-states.

Lemma 5 i) There exists $\bar{\alpha}>0$, such that there is no interior steady-states if $\alpha \leq \bar{\alpha}$. ii) There exists $\hat{\alpha}>\bar{\alpha}$, such that there exist interior steady-states if 
$\alpha \geq \hat{\alpha}$. iii) Upon existence, interior steady-states are locally unstable.

Proof. See the Appendix.

Lemma 5 shows the importance of labor productivity, or equivalently, of the level of wealth per capita, on the prevalence index in steady-state: there are thresholds below which there is an high prevalence and no prevention, and above which there is lower prevalence with some prevention. The intuition of this result hinges on the concavity and on the Inada condition imposed on the utility function: when the average production is low, resources are exclusively devoted to consumption since a marginal decrease of it produces a large desutility and since the marginal impact of prevention is independent of the level of productivity. Consequently, an interior steady-state is more likely to exist if the labor productivity is increased: the immediate marginal desutility of prevention (i.e. the LHS of (23)) is then lowered while its impact on future generations' discounted utility (i.e. the RHS of (23)) is increased.

Importantly, interior steady-states are locally unstable. As it will be done below, it is possible to characterize, under further conditions, saddle-path steadystates. Hence, from Lemmas 3 and 5, we conclude there are at most two kinds of paths that converge to a steady-state. First, we have a family of paths converging to corner steady-states. Second, upon existence of a saddle-path steadystate, we have the stable arm converging to one interior steady-state. All paths are candidates for optimality. We note that in related settings, the multiplicity 
of interior steady-states was established and studied in Delfino and Simmons [22], Goenka and Liu [32] and Goenka et al. [34]. In particular, Goenka et al. [34] give conditions for local uniqueness and show that complex dynamics can occurs. In an decentralized economy with endogenous growth, Goenka and Liu [33] show the existence of multiple steady-state that feature either sustained growth or poverty trap. The optimal health policy is larger in the former than in the latter.

Suppose now there exists at least one saddle-path steady-state. Let us compare the unique path that converges to this steady-state to the family of those which converge to the corner steady-state.

Lemma 6 Suppose there exists a saddle-path interior steady-state, denoted by $(\bar{a}, \bar{h})$. The stable arm that converges to $(\bar{a}, \bar{h})$ may not be optimal.

Proof. See the Appendix.

Lemma 6 shows that the existence of an interior steady-state does not implies that the stable arm is necessarily optimal. In the proof, we show that using a particular case where the long run cost of prevention is higher that the benefit in terms of production of having a lower share of infected individuals. Then, there exist sets of initial condition such that the intertemporal utility yield by the stable arm is lower than the one yield by the path converging to the corner steady-state. Conversely, there exists initial conditions, upon which the saddlepath is always preferred to those converging to the corner steady-state. 


\subsection{The global dynamics of prevention}

We now propose a geometrical representation of the results that have been previously established by drawing the phase diagram associated to the system of equations (18). To reduce the length of the proofs, an additional set of restrictions is assumed:

H5. $g_{12}^{\prime \prime}(h, a)=0$, and $-u^{\prime \prime \prime}(c) / u^{\prime \prime}(c) \geq-u^{\prime \prime}(c) / u^{\prime}(c)$.

Assumption H5 is sufficient to ensure that isocline $\dot{h}=0$, derived from function $\Phi$ given in equation (19) is well defined for $g>0$. The disease dynamics considered in Assumption H1 is now constrained by further assumptions on the impact of prevention on $\dot{a}_{t}$ : it is now convex and proportional to $a_{t}$. Moreover, the utility function restricts to a representative individual with absolute risk aversion lower than absolute prudence, a property satisfied by standard utility functions including those with harmonic absolute risk aversion. We established the following properties.

Lemma 7 i) The $\dot{a}=0$ locus is downward slopping in the plane $(a, h)$ and is such that $\dot{a}>0$ below the locus. ii) For $g>0$, the $\dot{h}=0$ locus is defined by a function $h=\chi(a)$ that satisfies $\lim _{a \rightarrow 0} \chi(a)=-\infty$ and is such that $\dot{h}>0$ above the locus. iii) As a increases, the $\dot{h}=0$ moves upward in $\left\{(a, h) \in \mathbb{R}^{2}, g(h, a)>0\right\}$.

Proof. See the Appendix. 
Lemma 7 gives enough information to draw the phase diagram associated to system (18). Depending on the existence of interior steady-states, optimal paths can hence be represented. Possible phase diagrams are given by figure 3a if there is no interior steady-states and by figure $3 \mathrm{~b}$ if there are two interior steady-states.

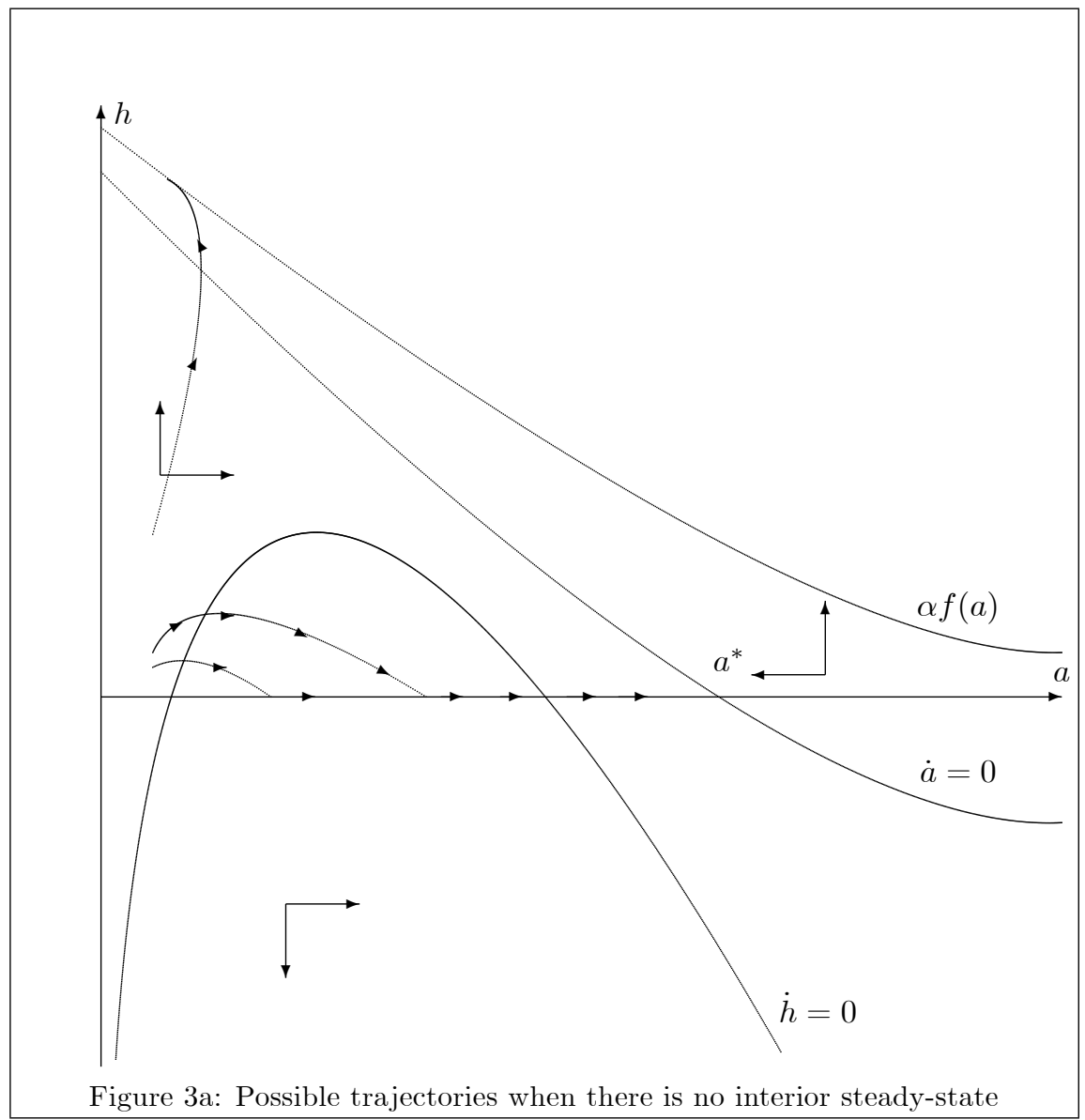

Note that we have represented the upper limit for $h$, given by function $\alpha f(a)$, above the $\dot{a}=0$ locus, but it as well can be below for some value of $a$. For an initial condition $a_{0} \in\left(0, a^{*}\right)$, there is hence a family of feasible paths: they converge toward the corner steady-state $\left(0, a^{*}\right)$ and provided that the $\dot{h}=0$ locus is above the horizontal axis, a positive level of prevention is possible for 
a finite interval of time. The diffusion of the disease may be slowed down for a while but, ultimately, the prevalence index reaches the long-run level with no intervention. Another family of paths is drawn in Figure 3a: they move to the vertical axis with a high level of prevalence. These paths are however not feasible since the prevention monotonously increases with time and reaches in finite time the upper limit given by $\alpha f(a)$. The consumption is there equal to zero and, consequently, the path is not optimal.

Labor productivity, which has been shown in the previous section to be crucial for the existence of interior steady-states, has an impact on the dynamics. Geometrically, as $\alpha$ increases, the $\dot{h}=0$ locus and the constraint $\alpha f(a)$ move up. Since the $\dot{a}=0$ locus is left unchanged, interior steady-states are more likely to appear, as stated in lemma 5 and drawn in Figure $3 \mathrm{~b}$.

Two interior steady-states are represented in the phase diagram of Figure 3b: a first one with a higher level of prevention and a lower prevalence, which is saddle-path and a second one which is a repulsive cycle. Hence, in addition to the families of paths that have been considered in the case without interior steady-states, there is a unique path that converges to the saddle-path steadystate. Remark that if there exist more than two interior steady-states, the phase diagram would exhibit alternatively saddle-paths and cycles. If the stable arm converging to the steady-state $(\bar{h}, \bar{a})$ is optimal, the prevention monotonically increase with time if $a_{0}<\bar{a}$ and can be an hump shaped function of time if $a_{0}>\bar{a}$. 


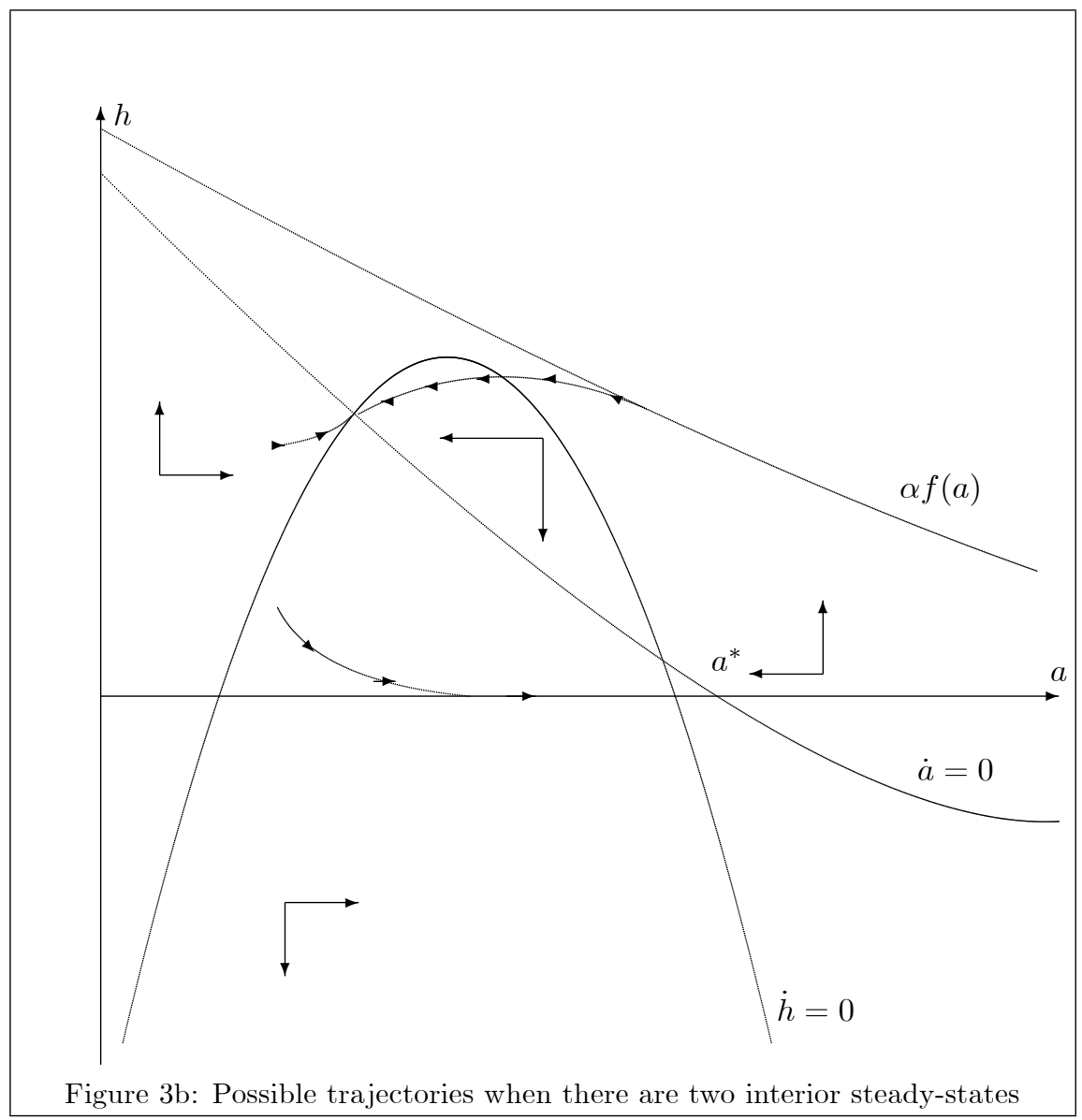

In this phase diagram analysis, we have insisted on the fact that on the neighborhood of the vertical axis, the growth rate of prevention is always positive (i.e. $\dot{h}>0$ ). This behavior is induced, using (18), by the assumption of a "not too concave" relationship between the infectious disease growth rate and the prevention. More precisely, it is true if:

$$
\lim _{h \rightarrow 0} \frac{g_{11}^{\prime \prime}(h, 0)}{g_{1}^{\prime}(h, 0)}<-\frac{u^{\prime \prime}(\alpha f(0))}{u^{\prime}(\alpha f(0))}
$$

which is satisfied in Assumption H5. 
Note finally that any path that may converge to the vertical axis, and thus to the elimination of the infectious disease, necessarily reaches in finite time the resource constraint and is therefore not optimal. Assuming the opposite inequality than that of (24) would not change the statement about the impossibility to eliminate, but simply the phase diagram. In the next section, we assume that $a_{\min }>0$, which allows to consider the elimination of the infectious disease.

\section{Elimination of infectious diseases}

Lemma 4 has shown that elimination was not possible in the case a $a_{\min }=0$. We now consider the case with a positive threshold below which the infectious disease remains constant at a negligible prevalence rate. Below, we first show that a trajectory that converges to $a_{\min }>0$ is feasible and then give the conditions for its optimality.

\subsection{The trajectory toward elimination}

We start with the following result.

Lemma 8 An optimal path that converges to the corner steady-state $\left(a_{\min }, 0\right)$ may exist. It satisfies $h_{t}=0$ for $t$ large enough.

Proof. See the Appendix.

If $a_{\min }>0$ is allowed, the elimination is feasible in our model. Since $a_{\min }$ can be reached in finite time, $h_{t}$ also reaches zero in finite time, as prevention has 
no impact when $a_{t}=a_{\min }$.

\subsection{Global dynamics with elimination}

Let us now see how our phase diagrams are modified: a new feasible path appears, which yields to the elimination of the infectious disease in finite time.

Figure 4 a represents a possible phase diagram when there is no interior steadystate.

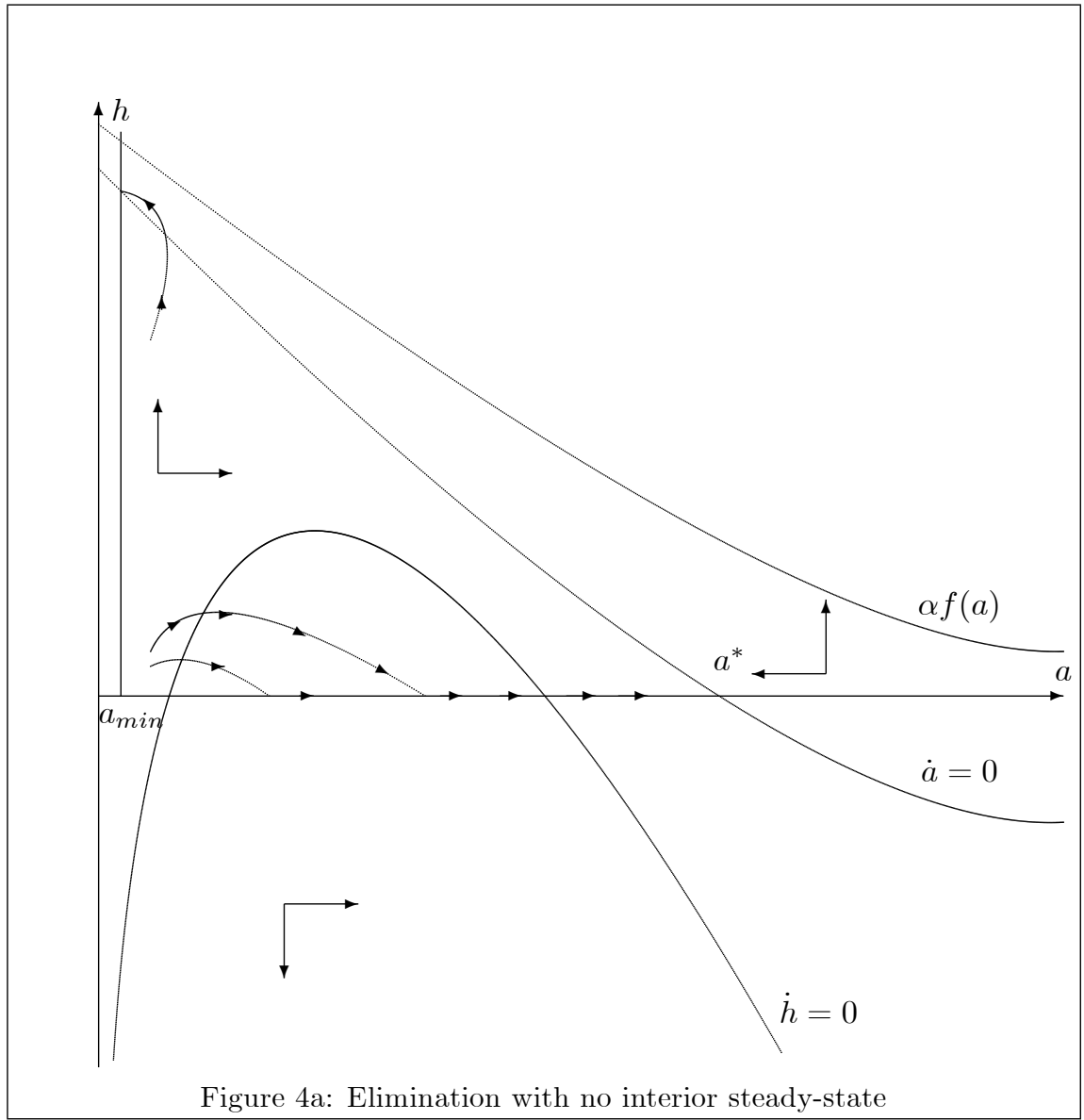

The family of paths that converges to the corner steady-state $\left(0, a^{*}\right)$ is still represented in Figure 4a. Moreover, there is a path that reaches the vertical axis in finite time, along which prevention monotonically increases. Moreover, 
the path is unique as it necessarily goes through the intersection between the vertical line at $a_{\min }$ and the $\dot{a}=0$ locus. A necessary condition for the existence of such a path is simply that those coordinates are feasible, meaning that the $\breve{h}$ such that $g\left(\breve{h}, a_{\text {min }}\right)=0$ satisfies the following condition: $\breve{h}<f\left(a_{\min }\right)$. A path leading to elimination also appears when there are interior steady-states, as it is shown by Figure $4 \mathrm{~b}$.

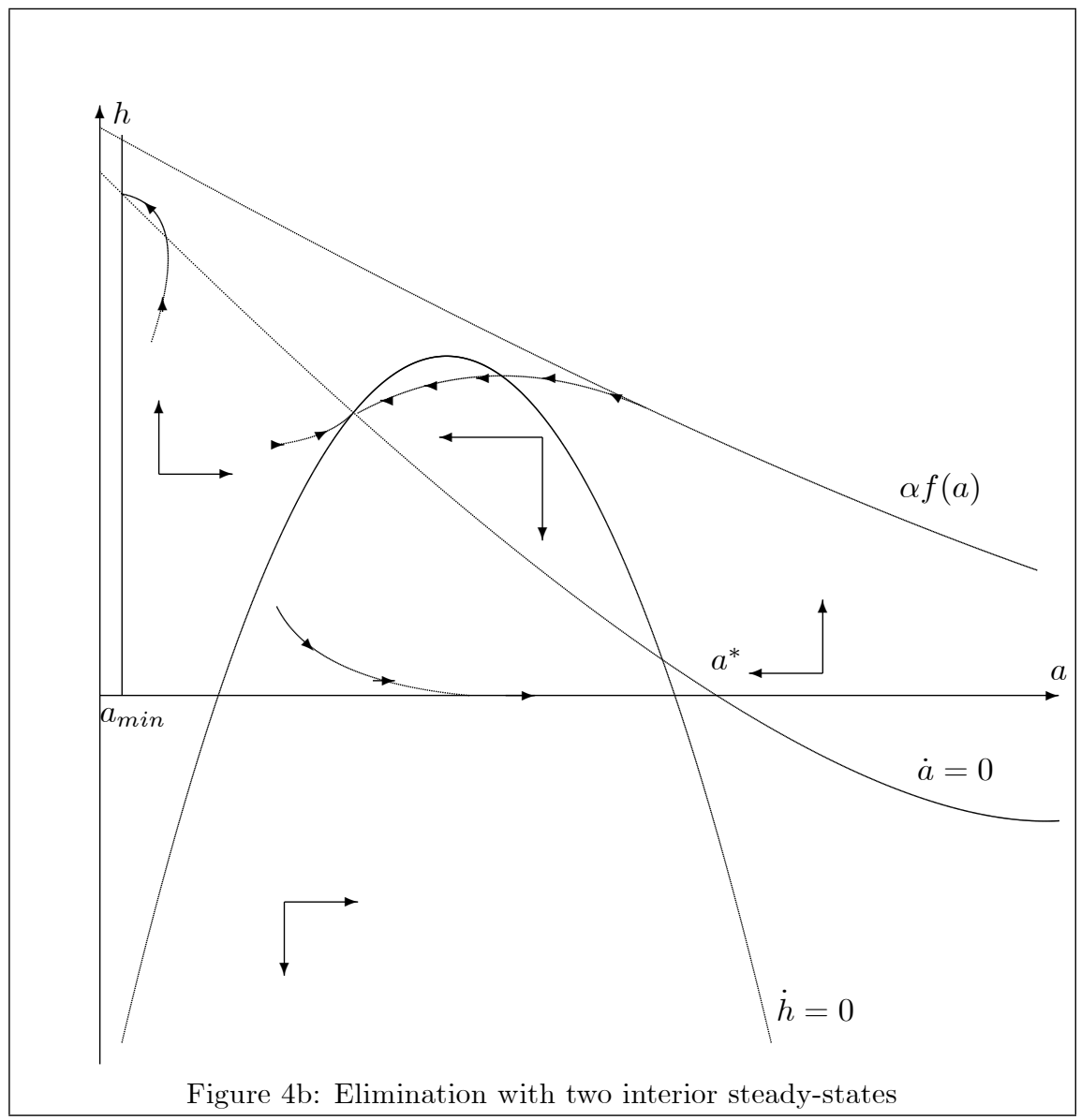

In the following lemma, we give a condition for the optimality of the elimination of the infectious disease.

Lemma 9 Suppose there exists a path that leads to the elimination of the in- 
fectious disease. There is a threshold for the pure discount rate, denoted $\bar{\rho}$, such that for $\rho<\bar{\rho}$, this path is optimal.

Proof. See the Appendix.

When elimination is achieved, the discounted utility of generation $t$, which is given by $u\left(c_{t}\right) / \theta\left(a_{t}\right)$, is the highest possible since there is no prevention and the prevalence index is zero. This however implies that generations that have lived before the elimination have supported large reduction of their welfare due to the necessarily high levels of prevention devoted to the control of the disease. The path that yields to elimination is thus optimal if the pure discount rate is sufficiently small. In a rather different setting, Barrett and Hoel [5] show that elimination is optimal if the exogenous cost of the control is not too large. In our setting, the cost is endogenous and satisfies a budget equation. Our result rather hinges on the comparison of intertemporal utilities. By comparing intertemporal profits of obtained by various vaccination strategies, Geoffard and Philipson [31] also show that the discount rate is a key variable but they show that elimination is preferred if discounting is large.

\subsection{A hand solved example}

Let us illustrate our dynamics with a simple example that encompass the two cases described in Section 2. Concerning the infectious disease, we first assume that the over-mortality rate of infected persons is zero $(\gamma=0)$, which considerably simplifies the problem as $n^{\prime}(a)=0$. Moreover, to reduce the number 
of parameters, we assume $n(0)=0$, which simply means that the death rate equals the birth rate. While unrealistic, those assumptions are often made in theoretical works (see e.g. Rowthorn and Toxvaerd [46]). We notice that both restrictions are consistent with $\mathrm{H} 1$ and $\mathrm{H} 3$. We also suppose that the impact of prevention on the infectious contacts is linear by writing: $\sigma(h)=\sigma_{0}-h$, and assuming that $h \in\left[0, \sigma_{0}\right]$. The dynamics of the prevalence index is thus given by: $\dot{a}_{t}=a_{t}\left[\sigma_{0}-h_{t}-\zeta\left(1+a_{t}\right)\right]$ where $\zeta=\beta \pi$ for the model describing the HIV case and $\zeta=\delta+\beta$ for the model describing a case of flu. The endemic steady-state without control is thus $a^{*}=\sigma_{0} / \zeta-1$, which is positive provided that $\sigma_{0}>\zeta$. Concerning the economy, we assume $u(c)=\ln c$ and $f(a)=1-a$, which imposes $a \leq 1$.

The optimal control problem is thus:

$$
\begin{aligned}
& \int_{0}^{\infty} e^{-\rho t} \ln c_{t} d t \\
& \begin{array}{l|l}
\text { s.t. } & \dot{a}_{t}=\mid \begin{array}{ll}
a_{t}\left[\left(\sigma_{0}-\alpha-\zeta\right)+c_{t}-(\zeta-\alpha) a_{t}\right] & \text { if } a_{t}>a_{\min } \\
0 & \text { if } a_{t} \leq a_{\min }
\end{array} \\
\max \left\{0, \alpha-\alpha a_{t}-\sigma_{0}\right\} \leq c_{t} \leq \alpha-\alpha a_{t} & \\
a_{0} \in\left(a_{\min }, \min \left\{a^{*}, 1\right\}\right), \text { given. } &
\end{array}
\end{aligned}
$$

For $a_{t}>a_{\min }$, the optimal dynamics is given by the following system:

$$
\left\{\begin{array}{l}
\dot{c}_{t}=-c_{t}\left[\rho+(\zeta-\alpha) a_{t}\right] \\
\dot{a}_{t}=a_{t}\left[\left(\sigma_{0}-\alpha-\zeta\right)+c_{t}-(\zeta-\alpha) a_{t}\right]
\end{array}\right.
$$

while the optimal prevention is given by $h_{t}=\alpha\left(1-a_{t}\right)-c_{t}$.

Two different cases shall be considered $\alpha \leq \zeta$ and $\alpha>\zeta$. For $\alpha \leq \zeta$, the phase diagram is presented in Figure 5a. The diagram represents two lines: 
the isocline $\dot{a}_{t}=0$ and the line given by $c_{t}=\alpha\left(1-a_{t}\right)$, which represents the upper limit for $c_{t}$ and the situation such that $h_{t}=0$; the isocline $\dot{c}_{t}=0$ does not appear here as it is in the negative quadrant. In this diagram, there is thus no interior steady-state, which is consistent with Lemma 7 as the labor productivity, given by $\alpha$, is low. For an initial prevalence $a_{0}$, two trajectories are feasible, which converge to the corner steady-states, $a_{\min }$ and $a^{*}$. The first one features a temporary decline in consumption till the prevalence reaches $a_{\min }$; then, consumption jumps to its highest possible level. In this trajectory, prevention first increases with time and fall to zero when the infectious disease is eliminated (Lemma 8). We see that such a trajectory is not likely to be optimal if $a_{\min }=0$ as consumption would converge to zero (Lemma 4). The second trajectory features a infinite decline in consumption as the infectious disease converges to the endemic steady-state; the prevention is always zero in that case (Lemma 3). Among those two trajectories, one is optimal and Figure 5a is a nice illustration of Lemma 9. Compared to the second one, the trajectory that lead to elimination indeed imposes initially a lower consumption that is compensated later by a higher one. Such a strategy can be optimal only if the 
discount rate is low.

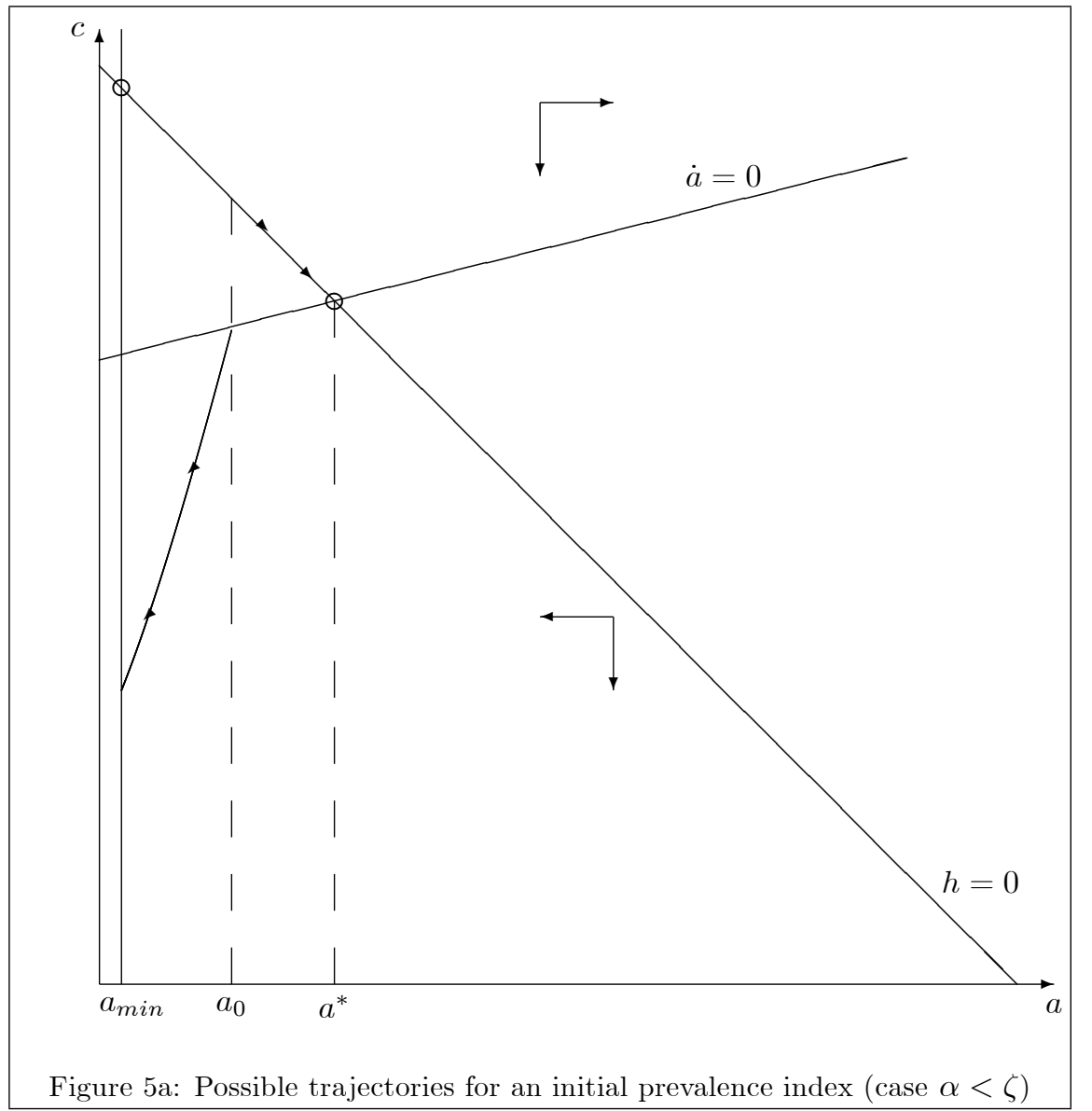

For $\alpha>\zeta$ (i.e. if the level of productivity is sufficiently large), the phase diagram analysis (Figure $5 \mathrm{~b}^{5}$ ) now represents the two isoclines $\dot{a}_{t}=0$ and $\dot{c}_{t}=0$ and the line $c_{t}=\alpha\left(1-a_{t}\right)$. We see there exists an interior steady-state, which is given by $\bar{a}=\rho /(\alpha-\zeta), \bar{c}=\alpha+\zeta-\rho-\sigma_{0}$, and $\bar{h}=\sigma_{0}-\zeta[1+\rho /(\alpha-\zeta)]$, and is saddle-path (as established in Lemma 5). We represented the trajectories for two initial prevalence index $a_{0}^{l}$ and $a_{0}^{h}$. The trajectory that converges to the interior steady-state features a consumption that decreases over time for the

\footnotetext{
${ }^{5}$ The diagram represents three lines: the isoclines $\dot{c}_{t}=0$ and $\dot{a}_{t}=0$ and the $c_{t}=\alpha-\alpha a_{t}$,
} which represents the upper limit for $c_{t}$ and the situation such that $h_{t}=0$. 
low initial prevalence and increase for the large one. There are, moreover, two additional trajectories that converge to $a_{\min }$ or $a^{*}$. The second corner steadystate can be reached only if the initial prevalence is larger than $\bar{a}$. As in the case with a low $\alpha$, the choice between those trajectories depends on the pure discount rate: the more the initial prevention, the higher the long run consumption.

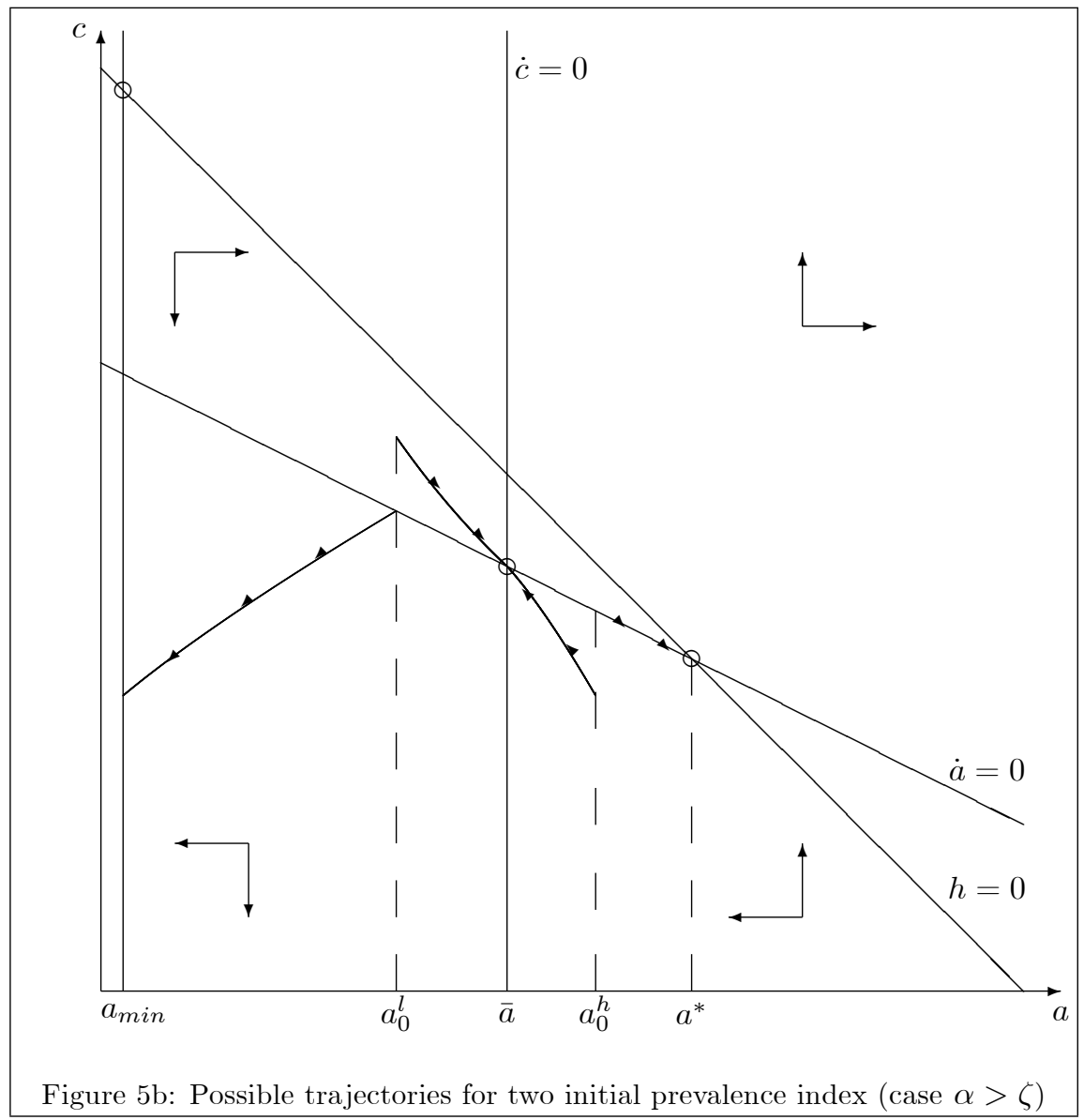

\section{Conclusion}

In this article, we have exhibited the relative role of resource constraints and individual preferences on the dynamic of the optimal prevention. Resources constraints are crucial for defining which paths are feasible, while preferences, 
and notably the discount rate, are used to characterize optimality. In the limit case of no pure discounting, as in Ramsey [45], "the-sooner-the-better" strategy is always optimal, provided there is an minimal threshold below which the infectious disease is considered as eliminated. This strategy is initially very costly in term of prevention but permits to set future expenses to zero. The is a theoretical ground for a "whatever it costs" response when economies face a new infectious disease.

Possible extensions of the present work may include the traditional decomposition of the population in three classes to include the infected people that recovered from illness, a production economy (as in Goenka et al. [34]) and/or some endogenous growth factors (Goenka and Liu [33]). The size of the dynamic system would then increase making the analytical resolution of the model more difficult. Future researches should also incorporate delay and age structure effects (Boucekkine et al. [12]) as they are crucial for most infectious diseases. Importantly, we aim at incorporating individual behaviors and decentralizing the optimum. The role of fiscal and debt policies, in particular, are in our research agenda. 


\section{Appendix}

Proof of Lemma 1. The program (17) is a non autonomous problem with endogenous discounting. Let us rewrite it as an exogenous discounting problem using the virtual time $\operatorname{method}^{6}$ notably used by Uzawa [48]. This method is based on a change of time scale, which can be applied as the state dynamics is autonomous. First define: $\delta_{t}=\int_{0}^{t} \theta\left(a_{s}\right) d s ;$ since $\delta$ is invertible from $\mathbb{R}_{+}$to $\mathbb{R}_{+}$, it is possible to characterize $t=\tau(\delta)$; moreover, $d \delta=\theta\left(a_{t}\right) d t$. According to Assumption H3, $\theta(a)>0$. The social planner's program (17) is then equivalent to:

$$
\begin{aligned}
& \max _{\tilde{h}_{\delta}} \int_{0}^{\infty} e^{-\delta} \frac{u\left(\alpha f\left(\tilde{a}_{\delta}\right)-\tilde{h}_{\delta}\right)}{\theta\left(\tilde{a}_{\delta}\right)} d \delta, \\
& \text { s.t. } \mid \begin{array}{l}
\frac{d \tilde{a}_{\delta}}{d \delta}=\mid \begin{array}{l}
\frac{g\left(\tilde{h}_{\delta}, \tilde{a}_{\delta}\right) \tilde{a}_{\delta}}{\theta\left(\tilde{a}_{\delta}\right)} \\
0 \quad \text { if } \tilde{a}_{\delta}>a_{\text {min }} \\
0 \leq \tilde{h}_{\delta} \leq \alpha f\left(\tilde{a}_{\delta}\right)
\end{array} \text { and } \tilde{a}_{\delta} \in\left(a_{\min }, a^{*}\right) \text { given. }
\end{array}
\end{aligned}
$$

where $\left(\tilde{a}_{\delta}, \tilde{h}_{\delta}\right) \equiv\left(a_{\tau(\delta)}, h_{\tau(\delta)}\right)$. Since there is no ambiguity, we will nevertheless keep the usual notations $\left(a_{t}, h_{t}\right)$. Let us denote $a\left(., t_{0}, a_{0}, h().\right)$ the unique solution of the state dynamics with initial condition $a_{0}$ at time $t_{0}$. Let $K=\left\{h(\right.$.$\left.) piecewise continuous such that 0 \leq h(t) \leq \alpha f\left(a\left(., t_{0}, a_{0}, h().\right)\right)\right\}$

The existence problem is then standard (Anița et al. [3]). Let us denote

$$
J\left(h, a^{h}\right)=\int_{0}^{\infty} e^{-t} \frac{u\left(\alpha f\left(a^{h}\right)-h\right)}{\theta\left(a^{h}\right)} d t
$$

\footnotetext{
${ }^{6}$ Francis and Kompas [26] propose a nice presentation of the method and of its conditions of application.
} 
where $a^{h}$ is the solution of

$$
\dot{a}=\mid \begin{array}{ll}
\frac{g(h, a) a}{\theta(a)} & \text { if } a>a_{\min } \\
0 & \text { if } a \leq a_{\min },
\end{array}
$$

and $a(0)=a_{0} \in\left(a_{\min }, a^{*}\right)$.

We start by proving that $K$ is convex. Let us consider $h_{1}$ and $h_{2}$ in $K$, then

$$
0 \leq h_{1} \leq \alpha f\left(h_{1}\right) \text { and } 0 \leq h_{2} \leq \alpha f\left(h_{2}\right) .
$$

Let us consider $\sigma \in[0,1]$ and $h=\sigma h_{1}+(1-\sigma) h_{2}$. Then

$$
0 \leq h \leq \alpha\left(\sigma f\left(h_{1}\right)+(1-\sigma) f\left(h_{2}\right)\right) .
$$

Concavity of $f$ then enables to end the proof of the convexity of $K$.

We now follow the three steps of the existence proof presented in Aniţa et al. ([3]) page 63 .

Observe first that the application $a \longmapsto g(h, a) a / \theta(a)$ is locally lipschitz for $a \in\left(a_{\min }, a^{*}\right)$ and that, given the assumptions on $g$, for any admissible control,

$$
\dot{a} \leq \frac{g\left(0, a_{\min }\right) a_{\min }}{\theta\left(a_{\min }\right)} .
$$

We conclude that: $a_{\min } \leq a^{h} \leq a_{0} e^{\frac{g\left(0, a_{\min }\right) a_{\min }}{\theta\left(a_{\min }\right)}}<a^{*}$. Moreover, according to the properties of $u, f$ and $\theta$, one has:

$$
0<\frac{u\left(\alpha f\left(a_{t}^{h}\right)-h_{t}\right)}{\theta\left(a_{t}^{h}\right)}<\frac{u\left(\alpha f\left(a_{t}^{h}\right)\right)}{\theta\left(a_{\min }\right)}<\frac{u\left(\alpha f\left(a_{\min }\right)\right)}{\theta\left(a_{\min }\right)}
$$

from which we deduce that $\max _{h \in K} J\left(h, a^{h}\right)$ is positive and finite.

Let

$$
d=\sup _{h \in K} J\left(h, a^{h}\right) .
$$


For $n \in \mathbb{N}^{*}$, there exists $h_{n} \in K$ such that

$$
d-\frac{1}{n} \leq J\left(h-n, a^{h_{n}}\right) \leq d
$$

As $K$ is bounded, there exists a subsequence $\left\{h_{n_{k}}, a^{h_{n_{k}}}\right\}$ such that $\left(h_{n_{k}}, a^{h_{n_{k}}}\right)$ converges weakly to $(\tilde{h}, \tilde{a})$ is $L^{2}$. As $K$ is a closed convex set, then $\tilde{h} \in K$. As $a^{h_{n_{k}}}$ is bounded, $\left(a^{h_{n_{k}}}\right)$ converges to $\tilde{a}$ in $\mathcal{C}$.

Let us now establish the necessary conditions.

Let $\mathcal{H}\left(h_{t}, a_{t}, \mu_{t}\right)$ be the current value of the Hamiltonian associated with problem (27) that is given by:

$$
\mathcal{H}\left(h_{t}, a_{t}, \mu_{t}\right)=\frac{u\left(\alpha f\left(a_{t}\right)-h_{t}\right)}{\theta\left(a_{t}\right)}+\mu_{t} \frac{g\left(h_{t}, a_{t}\right) a_{t}}{\theta\left(a_{t}\right)},
$$

and $\mu_{t}$ be the associated costate variable. Denoting by $p$ and $q$ the Lagrange multipliers associated with the inequality constraints, the Lagrangian in the current value is written as:

$\mathcal{L}\left(h_{t}, a_{t}, \mu_{t}, p_{t}, q_{t}\right)=\frac{u\left(\alpha f\left(a_{t}\right)-h_{t}\right)}{\theta\left(a_{t}\right)}+\mu_{t} \frac{g\left(h_{t}, a_{t}\right) a_{t}}{\theta\left(a_{t}\right)}+p_{t} h_{t}+q_{t}\left(\alpha f\left(a_{t}\right)-h_{t}\right)$.

Let us consider an optimal pair $(\tilde{a}, \tilde{h})$. According to the previous remark and applying the maximum principle (Grass et al. [35], Proposition 3.52), there exists a continuous function $\mu$, which is piecewise continuously differentiable, and two piecewise continuous multipliers $\left(q_{t}, p_{t}\right)$ that satisfy:

$$
\mathcal{H}\left(\tilde{h}_{t}, \tilde{a}_{t}, \mu_{t}\right)=\max _{h_{t \in K}} \mathcal{H}\left(h_{t}, \tilde{a}_{t}, \mu_{t}\right)
$$


Notice that the condition $\lim _{c \rightarrow 0} u^{\prime}(c)=+\infty$ prevents constraint $h_{t}=\alpha f\left(a_{t}\right)$ to be binding. Extrema of $\mathcal{H}\left(h_{t}, \tilde{a}_{t}, \mu_{t}\right)$ for $h_{t} \in K$ satisfy

$$
-\frac{u^{\prime}\left(\alpha f\left(a_{t}\right)-h_{t}\right)}{\theta\left(a_{t}\right)}+\mu_{t} \frac{g_{1}^{\prime}\left(h_{t}, a_{t}\right)}{\theta\left(a_{t}\right)} a_{t}+p_{t}=0,
$$

together with the slackness condition:

$$
p_{t} h_{t}=0, p_{t} \geq 0, h_{t} \geq 0 .
$$

According to Assumptions $\mathrm{H} 1$ and $\mathrm{H} 4$, if the interior solution is an extrema, it is necessary that $\mu_{t} \leq 0$. As the second order derivative yields

$$
\frac{u^{\prime \prime}\left(\alpha f\left(a_{t}\right)-h_{t}\right)}{\theta\left(a_{t}\right)}+\mu_{t} \frac{g_{11}^{\prime \prime}\left(h_{t}, a_{t}\right)}{\theta\left(a_{t}\right)} a_{t}
$$

and using Assumptions $\mathrm{H} 1$ and $\mathrm{H} 4$, if the interior solution is an extrema, it is a maximum.

Pontryagin's Maximum principle also requires at every time $t$ where $h$ is continuous:

$$
\begin{aligned}
\dot{\mu}_{t}= & -\alpha f^{\prime}\left(a_{t}\right) \frac{u^{\prime}\left(\alpha f\left(a_{t}\right)-h_{t}\right)}{\theta\left(a_{t}\right)}-\mu_{t}\left(\frac{g_{2}^{\prime}\left(h_{t}, a_{t}\right) a_{t}+g\left(h_{t}, a_{t}\right)}{\theta\left(a_{t}\right)}-1\right) \\
& -q_{t} \alpha f^{\prime}\left(a_{t}\right)+\frac{\theta^{\prime}\left(a_{t}\right)}{\theta(a)} \mathcal{H}\left(h_{t}, a_{t}, \mu_{t}\right)
\end{aligned}
$$

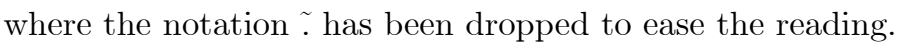

Finally, the transversality condition (see Michel [41]) is:

$$
\lim _{t \rightarrow \infty} e^{-t} \mathcal{H}\left(h_{t}, a_{t}, \mu_{t}\right)=0
$$


Proof of Lemma 2. For $h_{t}>0, p_{t}=0$. The expression for $\dot{h}_{t}$ is obtained by differentiating (40) with respect to $t$ and rearranging using (43). For $h_{t}=0$, the derivation of the system is immediate.

Proof of Lemma 3. In the neighborhood $V$ of $\left(a^{*}, 0\right)$ the interior solution of $h_{t}$ solves:

$\left.\dot{h}_{t}\right|_{\left(a^{*}, 0\right)}=\frac{-\left[g_{2}^{\prime}\left(0, a^{*}\right) a^{*}-\theta\left(a^{*}\right)\right]+\left[-\alpha f^{\prime}\left(a^{*}\right)+\frac{u\left(\alpha f\left(a^{*}\right)\right)}{u^{\prime}\left(\alpha f\left(a^{*}\right)\right)} \frac{\theta^{\prime}\left(a^{*}\right)}{\theta\left(a^{*}\right)}\right] g_{1}^{\prime}\left(0, a^{*}\right) a^{*}}{-\theta\left(a^{*}\right)\left[\frac{u^{\prime \prime}\left(\alpha f\left(a^{*}\right)\right)}{u^{\prime}\left(\alpha f\left(a^{*}\right)\right)}+\frac{g_{11}^{\prime \prime}\left(0, a^{*}\right)}{g_{1}^{\prime}\left(0, a^{*}\right)}\right]}$.

Thus, $\left.\dot{h}_{t}\right|_{\left(a^{*}, 0\right)}$ can be positive or negative, depending on the exogenous values of $\left(\alpha, a^{*}\right)$. If $\left.\dot{h}_{t}\right|_{\left(a^{*}, 0\right)}>0$, the constraint $h_{t} \geq 0$ implies $h_{t}=0$. If $\left.\dot{h}_{t}\right|_{\left(a^{*}, 0\right)}<0$, the trajectory reaches $h=0$ in finite time $t_{0}$ and then $h_{t}=0$ for all $t \geq t_{0}$.

Proof of Lemma 4. In the neighborhood $V$ of $(0, h), c>0$ and $h$ is finite, the interior solution of $\left(a_{t}, h_{t}\right)$ solves:

$$
\left.\dot{h}_{t}\right|_{(0, h)}=\frac{1}{-\left[\frac{u^{\prime \prime}(\alpha f(0)-h)}{u^{\prime}(\alpha f(0)-h)}+\frac{g_{1}^{\prime \prime}(h, 0)}{g_{1}^{\prime}(h, 0)}\right]},
$$

which given $\mathrm{H} 1$ and $\mathrm{H} 4$ is positive. Due to $\dot{a}_{t}=g\left(h_{t}, a_{t}\right) a_{t}, a=0$ is not achieved in finite time. Thus the constraint $h=\alpha f(0)$ is reached in finite time. According to lemma 2, this solution is not optimal.

Proof of Lemma 5. As a preliminary, use (20) as an implicit equation to define $h=\eta(a)$, which, given Assumption H1, satisfies $\eta^{\prime}(a)<0$ and $\eta\left(a^{*}\right)=0$, and 
replace it in (21) to define the function $\phi(a, \alpha)$ such that:

$$
\begin{aligned}
\phi(a, \alpha)= & -\left[g_{2}^{\prime}(\eta(a), a) a-\theta(a)\right] \\
+ & {\left[-\alpha f^{\prime}(a)+\frac{u(\alpha f(a)-\eta(a))}{u^{\prime}(\alpha f(a)-\eta(a))} \frac{\theta^{\prime}(a)}{\theta(a)}\right] g_{1}^{\prime}(\eta(a), a) a }
\end{aligned}
$$

Function $\phi(a, \alpha) \in C^{2}\left(D(\alpha) \times \mathbb{R}^{+}, \mathbb{R}\right)$ where:

$$
D(\alpha)=\left\{a \in \mathbb{R}^{+} / \alpha f(a)-\eta(a)>0\right\} .
$$

Then, an interior steady-state is a $a \in\left(a^{*}, 0\right)$ such that $\phi(a, \alpha)=0$.

To prove claim $i$ ), use Lemma 2 and Assumption H4 to establish the following limit:

$$
\lim _{\alpha \rightarrow 0} \phi(a, \alpha)=-\left[g_{2}^{\prime}(0, a) a-\theta(a)\right]>0,
$$

and conclude using the continuity of $\phi(.,$.$) with respect to \alpha$.

Claim ii): since $\alpha \longmapsto \alpha f(a)$ is strictly increasing and since $\lim _{\alpha \rightarrow \infty} \alpha f(a)=$ $\infty$, there exists $\alpha$ such that $D(\dot{\alpha})=\mathbb{R}^{+}$. Suppose $\alpha \geq \dot{\alpha}$. Under Assumptions $\mathrm{H} 1$ and $\mathrm{H} 4, \phi(a, \alpha)$ decreases with $\alpha, \phi(0, \alpha)=\theta(0)>0$ and:

$$
\begin{aligned}
\phi\left(a^{*}, \alpha\right)= & -\left[g_{2}^{\prime}\left(0, a^{*}\right) a^{*}-\theta\left(a^{*}\right)\right] \\
& +\left[-\alpha f^{\prime}\left(a^{*}\right)+\frac{u\left(\alpha f\left(a^{*}\right)\right)}{u^{\prime}\left(\alpha f\left(a^{*}\right)\right)} \frac{\theta^{\prime}\left(a^{*}\right)}{\theta\left(a^{*}\right)}\right] g_{1}^{\prime}\left(0, a^{*}\right) a^{*},
\end{aligned}
$$

is negative for sufficiently large $\alpha$.

Claim iii): the stability property is obtained by computing the trace of the Jacobian matrix of system (18) on the neighborhood of any interior steady- 
state. Since:

$$
\begin{aligned}
\left.\frac{\partial \Phi\left(h_{t}, a_{t}\right)}{\partial h_{t}}\right|_{\Phi\left(h_{t}, a_{t}\right)=0} & =1-\frac{g_{2}^{\prime}\left(h_{t} a_{t}\right) a_{t}}{\theta\left(a_{t}\right)}, \\
\left.\frac{\partial g\left(h_{t}, a_{t}\right) a_{t}}{\partial a_{t}}\right|_{g\left(h_{t}, a_{t}\right)=0} & =\frac{g_{2}^{\prime}\left(h_{t} a_{t}\right) a_{t}}{\theta\left(a_{t}\right)},
\end{aligned}
$$

the trace is consequently equal to 1 . The steady-states are not stable.

Proof of Lemma 6 . Let us denote by $(\bar{a}, \bar{h})$ the interior steady-state and by $\left(a^{*}, 0\right)$ the corner steady-state. To prove the lemma, we compute the intertemporal utilities for each paths in a particular case.

Suppose that $a_{0}=\bar{a}<a^{*}$. Consider two paths that are candidates for optimality: the first one is given by: $h_{t}=\bar{h}$ and $a_{t}=\bar{a}$, and the second one is given by $h_{t}=0$ and $a_{t}=\hat{a}(\bar{a}, t)$ (where according to notations used in the proof of Lemma $1 \hat{a}(\bar{a}, t)=a(t, 0, \bar{a}, 0))$. The intertemporal utility yield by the first path is:

$$
\int_{0}^{\infty} e^{-t} \frac{u(\alpha f(\bar{a})-\bar{h})}{\theta(\bar{a})} d t=\frac{u(\alpha f(\bar{a})-\bar{h})}{\theta(\bar{a})} .
$$

The intertemporal utility yield by the second path is denoted $U(\bar{a})$ and satisfies:

$$
U(\bar{a})=\int_{0}^{\infty} e^{-t} \frac{u(\alpha f(\hat{a}(\bar{a}, t)))}{\theta(\hat{a}(\bar{a}, t))} d t .
$$

As $g(0, a) a$ is $\mathcal{C}^{2}$ according to Assumption $\mathrm{H} 1, \hat{a}(\bar{a}, t)$ has a derivative according to $\bar{a}$ and

$$
\begin{aligned}
& \frac{d}{d \bar{a}}\left(\frac{u(\alpha f(\hat{a}(\bar{a}, t)))}{\theta(\hat{a}(\bar{a}, t))}\right) \\
= & \frac{d \hat{a}(\bar{a}, t)}{d \bar{a}}\left(\frac{\alpha f^{\prime}(\hat{a}(\bar{a}, t)) u^{\prime}(\alpha f(\hat{a}(\bar{a}, t)))}{\theta(\hat{a}(\bar{a}, t))}-\frac{\theta^{\prime}(\hat{a}(\bar{a}, t)) u(\alpha f(\hat{a}(\bar{a}, t)))}{\theta^{2}(\hat{a}(\bar{a}, t))}\right)<0
\end{aligned}
$$


Since $U^{\prime}(\bar{a})<0$, conclude that $U(\bar{a})>U\left(a^{*}\right)$. Hence, the first path is not optimal if:

$$
\frac{u(\alpha f(\bar{a})-\bar{h})}{\theta(\bar{a})}<\frac{u\left(\alpha f\left(a^{*}\right)\right)}{\theta\left(a^{*}\right)},
$$

It is easy to check that the inequality in (55) may not be satisfied for a pair $(\bar{h}, \bar{a})$ that satisfies $(20)$ and $(21)$.

Proof of Lemma 7. We consider successively the $\dot{a}=0$ locus and the $\dot{h}=0$ locus.

Claim i). The $\dot{a}=0$ locus for all $a>0$ is given by the implicit function $g(h, a)=0$. Given Assumption H1, the locus is downward slopping in the plane $(a, h)$, and is such that $\dot{a}>0$ below the locus and $\dot{a}<0$ above.

Claim ii). Using the definition of $\dot{h}$ in (18) and Assumption H5, the $\dot{h}=0$ locus is given by the function $\psi(h, a, \alpha)=0$ where:

$$
\begin{aligned}
\psi(h, a, \alpha)= & {\left[-\alpha f^{\prime}(a) \frac{u^{\prime \prime}(\alpha f(a)-h)}{u^{\prime}(\alpha f(a)-h)}+\frac{\theta^{\prime}(a)}{\theta(a)}\right] g(h, a) a } \\
& +\left[-\alpha f^{\prime}(a)+\frac{u(\alpha f(a)-h)}{u^{\prime}(\alpha f(a)-h)} \frac{\theta^{\prime}(a)}{\theta(a)}\right] g_{1}^{\prime}(h, a) a \\
& -g_{2}^{\prime}(h, a) a+\theta(a), \\
\psi_{1}^{\prime}(h, a, \alpha)= & {\left[-\alpha f^{\prime}(a) \frac{u^{(3)}(\alpha f(a)-h) u^{\prime}(\alpha f(a)-h)+\left(u^{(2)}(\alpha f(a)-h)\right)^{2}}{\left(u^{\prime}(\alpha f(a)-h)\right)^{2}}\right] g(h, a) a } \\
& +\frac{\theta^{\prime}(a)}{\theta(a)}\left[\frac{u(\alpha f(a)-h) u^{(2)}(\alpha f(a)-h)}{\left(u^{\prime}(\alpha f(a)-h)\right)^{2}}\right] g_{1}^{\prime}(h, a) a \\
+ & {\left[-\alpha f^{\prime}(a)+\frac{u(\alpha f(a)-h)}{u^{\prime}(\alpha f(a)-h)} \frac{\theta^{\prime}(a)}{\theta(a)}\right] g_{11}^{\prime}(h, a) a }
\end{aligned}
$$

which, using Assumptions H1, $\mathrm{H} 4$ and $\mathrm{H} 5$, satisfies $\psi_{1}^{\prime}(h, a, \alpha)>0$ for all $(h, a)$ 
below the $\dot{a}=0$ locus (i.e. for $g(h, a)>0$ ). It is then possible to define the function $\chi$ such that $h=\chi(a, \alpha)$, which satisfies: $\lim _{a \rightarrow 0} \chi(a, \alpha)=-\infty$.

Using (18), compute, on the neighborhood of $(0,0)$, the interior solution of $h_{t}$ to obtain:

$$
\left.\dot{h}_{t}\right|_{(0,0)}=-\left[\frac{u^{\prime \prime}(\alpha f(0))}{u^{\prime}(\alpha f(0))}+\frac{g_{11}^{\prime \prime}(0,0)}{g_{1}^{\prime}(0,0)}\right]^{-1},
$$

which is positive given the convexity of $g$ (Assumption H5). Conclude that $\dot{h}>0$ above the locus and that $\dot{h}<0$ below.

Claim iii). Define $R=\left\{(a, h) \in \mathbb{R}^{2}, g(h, a)>0\right\}$. Using Assumptions H1, $\mathrm{H} 4$ and $\mathrm{H} 5$, we have $\psi_{3}^{\prime}(h, a, \alpha)<0$ for all $(h, a)$ such that $g(h, a)>0$. As $\psi_{1}^{\prime}(h, a, \alpha)>0$ on $R$, the claim is proved.

Proof of Lemma 8. In the neighborhood $V$ of $\left(a_{\min }, h\right), c>0$ and $h$ is finite, the interior solution of $\left(a_{t}, h_{t}\right)$ solves:

$$
\left.\left.\dot{h}_{t}\right|_{\left(a_{\min }, h\right)} \simeq \dot{h}_{t}\right|_{(0, h)}=\frac{1}{-\left[\frac{u^{\prime \prime}(\alpha f(0)-h)}{u^{\prime}(\alpha f(0)-h)}+\frac{g_{11}^{\prime \prime}(h, 0)}{g_{1}^{\prime}(h, 0)}\right]}>0,
$$

which given $\mathrm{H} 1$ and $\mathrm{H} 4$ is positive. As the optimal trajectory $(a, h)$ reaches $a_{\min }$ in finite time $t_{1}$, for $t>t_{1}, a(t)=a_{\min }$, which does not depends on $h$. Thus, for $t>t_{1}, h=0$.

Proof of Lemma 9. A path that leads to elimination in a finite time $T$ can be characterized by $\left\{h_{t}^{e}, a_{t}^{e}\right\}$ for all $t<T$ and by $h_{t}=0$ and $a_{t}=a_{\min }$ for all $t \geq 0$. This yields the following intertemporal utility:

$$
\int_{0}^{T} e^{-t} \frac{u\left(\alpha f\left(a_{t}^{e}\right)-h_{t}^{e}\right)}{\theta\left(a_{t}^{e}\right)} d t+e^{-T} \frac{u\left(\alpha f\left(a_{\min }\right)\right)}{\theta\left(a_{\min }\right)} .
$$


For $a_{\min }>0$ but small, then $\theta\left(a_{\min }\right)$ is close to zero, and therefore the intertemporal utility is very large while intertemporal utilities yield by any other path is smaller. We conclude by continuity. 


\section{References}

[1] L. J. Allen and G. E. Lahodny Jr, Extinction thresholds in deterministic and stochastic epidemic models, Journal of Biological Dynamics 6(2), (2012) 590-611.

[2] F. E. Alvarez, D. Argente, and F. Lippi, A simple planning problem for COVID-19 lockdown, NBER Working Paper No. 26981, 2020

[3] S. Aniţa, V. Arnăutu and V. Capasso, An Introduction to Optimal Control Problems in Life Sciences and Economics, Birkhäuser, 2011.

[4] C. Avery, W. Bossert, A. Clark, G. Ellison, and S. Fisher Ellison, Policy implications of models of the spread of Coronavirus: Perspectives and opportunities for economists, NBER Working Paper No. 27007, 2020

[5] S. Barrett and M. Hoel, Optimal disease eradication, Environment and Development Economics 12, (2007) 627-652.

[6] C. Bell and H. Gersbach, Growth and enduring epidemic diseases, Journal of Economic Dynamics and Control 37 (2013) 2083-2103.

[7] H. Benhcke, Optimal control of deterministic epidemics, Optimal Control Applications and Methods 21, (2000) 269-285.

[8] D. Bernoulli, Essai d'une nouvelle analyse de la mortalité causée par la petite vérole et des avantages de l'inoculation pour la prévenir, Memoire de mathématiques et de physique, Académie Royale des Sciences (1760) 1-45.

[9] Z. A. Bethune and A. Korinek, Covid-19 infection externalities: Trading off lives vs. livelihoods, NBER Working Paper No. 27009, 2020.

[10] D. E. Bloom and D. Canning, Epidemics and economics, PGDA working paper No. 9, Harvard School of Public Health.

[11] S. Bosi and D. Desmarchelier, Biodiversity, infectious diseases, and the dilution effect. Environmental Modeling \& Assessment 25 (2020), 277-292.

[12] R. Boucekkine, B. Diene and T. Azomahou, Growth economics of epidemics: A review of the theory, Mathematical Population Studies 15 (2008), $1-26$.

[13] R. Boucekkine, B. Diene and T. Azomahou, HIV/AIDS and development: A reappraisal of the productivity and factor accumulation effects, American Economic Review 106, (2016) 472-477.

[14] R. Boucekkine and J.-P. Laffargue, On the distributional consequences of epidemics, Journal of Economic Dynamics and Control 34 (2010) 231-245. 
[15] R. Boucekkine, B. Martínez and R. J. Ruiz-Tamarit, Optimal population growth as an endogenous discounting problem: The Ramsey case. In: Lecture Notes in Economics and Mathematical Systems, pp. 321-347, Springer, Cham, 2018

[16] T. Britton, Stochastic epidemic models: a survey. Mathematical biosciences 225(1), (2010) 24-35.

[17] B. Buonomo, P. Manfredi and A. d'Onofrio, Optimal time-profiles of public health intervention to shape voluntary vaccination for childhood diseases, Journal of Mathematical Biology 78, (2019) 1089-1113

[18] D. Cass, Optimum growth in an aggregative model of capital accumulation, Review of Economic Studies 32 (1965), 233-240.

[19] C. Castilho, Optimal control of an epidemic through educational campaigns, Electronic Journal of Differential Equation 125, (2006) 1-11.

[20] P. Corrigan, G. Glomm and F. Méndez, AIDS crisis and growth, Journal of Development Economics 77, (2005) 107-124.

[21] P. Dasgupta, On the concept of optimal population, Review of Economic Studies 36, (1969) 295-318.

[22] D. Delfino and P. J. Simmons, Positive and normative issues of economic growth with infectious disease, working paper, University of York, 2000.

[23] M. S. Eichenbaum, S. Rebelo and M. Trabandt, The macroeconomics of epidemics, NBER Working Paper No. 26882, 2020.

[24] G. Feichtinger, V. M. Veliov and T. Tsachev, Maximum principle for age and duration structured systems: A tool for optimal prevention and treatment of HIV, Mathematical Population Studies 11, (2004) 3-28.

[25] P. J. Francis, Optimal tax/subsidy combinations for the flu season, Journal of Economic Dynamics and Control 28, (2004) 2037-2054.

[26] J. Francis and T. Kompas, Uzawa's transformation and optimal control problems with variable rates of time preference, working paper, 1998.

[27] M. Gersovitz, Mathematical epidemiology and welfare economics, in P. Manfredi and A. D'Onofrio, Eds., Modeling the Interplay Between Human Behavior and the Spread of Infectious Diseases, Springer, 2013.

[28] M. Gersovitz and J. S. Hammer, The economical control of infectious diseases, Economic Journal 114, (2004) 1-27.

[29] M. Gersovitz and J. S. Hammer, Tax/subsidy policies toward vector-borne infectious diseases, Journal of Public Economics 89, (2005) 647-674. 
[30] P.-Y. Geoffard and T. Philipson, Rational epidemics and their public control, International Economic Review 37, (1996) 603-624.

[31] P.-Y. Geoffard and T. Philipson, Disease eradication: Private versus public vaccination, American Economic Review 87, (1997) 222-230.

[32] A. Goenka and L. Liu, Infectious diseases and endogenous fluctuations, Economic Theory 50, (2012) 125-149.

[33] A. Goenka and L. Liu, Infectious diseases, human capital and economic growth, Economic Theory 70, (2020) 1-47.

[34] A. Goenka, L. Liu and M. Nguyen, Infectious diseases and economic growth. Journal of Mathematical Economics 50, (2014) 34-53.

[35] D. Grass, J. P. Caulkins, G. Feichtinger, G. Tragler and D. A. Behrens, Optimal control of nonlinear processes with applications. In Drugs, Corruption and Terror, 2008 Springer-Verlag Berlin Heidelberg.

[36] H. W. Hethcote and J. A. Yorke, Gonorrhea transmission dynamics and control, Lecture Notes in Biomathematics 56, 1984. Springer-Verlag Berlin.

[37] W. O. Kermarck and A. G. Mac Kendrick, A contribution to the mathematical theory of epidemics, Proc. Royal Soc. London 115, (1927) 700-721.

[38] T. C. Koopmans, On the concept of optimal economic growth. In The Econometric Approach to Developpement Planning, North Holland, Amsterdam, 1965.

[39] M. Kremer, Integrating behavioral choice into epidemiological models of AIDS, Quarterly Journal of Economics 111, (1996) 549-573.

[40] R. M. May and R. M. Anderson, The transmission dynamics of Human Immunodeficiency Virus, in S. A. Levin, T. G. Hallam and L. J. Gross, Eds., Applied Mathematical Ecology, Springer-Verlag, Berlin, 1989.

[41] P. Michel, Some clarifications on the transversality condition, Econometrica 58, (1990) 705-723

[42] A. Momota, K. Tabata and K. Futagami, Infectious disease and preventive behavior in an overlapping generations model, Journal of Economic Dynamics and Control 29, (2005) 1673-1700.

[43] D. Morris, F., Rossine, J., Plotkin and S. Levin, Optimal, near-optimal, and robust epidemic control. Working paper. 2020. https://osf.io/rq5ct/

[44] R. Naresh, A. Tripathi and S. Omar, Modelling the spread of AIDS epidemic with vertical transmission, Applied Mathematics and Computation 178, (2006) 262-272. 
[45] F. P. Ramsey, A mathematical theory of savings, Economic Journal 38, (1928) 543-559.

[46] R. Rowthorn and F. Toxvaerd, The optimal control of infectious diseases via prevention and treatment, Cambridge Working Papers in Economics CWPE2027, 2020.

[47] S. P. Sethi, Optimal control of some simple deterministic epidemic models, The Journal of the Operational Research Society 29, (1978) 129-136.

[48] H. Uzawa, Time preference, the consumption function, and optimal asset holding, in J. N. Wolfe (ed.), Capital and Growth Papers in Honor of Sir John Hicks, The University of Edimburg Press, 485-504, 1968.

[49] P. van den Driessche, Reproduction numbers of infectious disease models. Infectious Disease Modelling 2(3), (2017) 288-303.

[50] K. H. Wickwire, Mathematical models for the control of pests and infectious diseases, A survey. Theoretical Population Biology 11, (1977) 182-238.

[51] A. Young, The gift of the dying: the tragedy of AIDS and the welfare of future African generations, Quarterly Journal of Economics 120, (2005) 423-466.

[52] J. Zhou and H.W. Hethcote, Population size dependent incidence in models for diseases without immunity. Journal of Mathematical Biology 32, (1994) 809-834. 